\title{
Attentional selection of objects or features: Evidence from a modified search task
}

\author{
JEFFREY R. W. MOUNTS \\ State University of New York, Geneseo, New York \\ and \\ ROBERT D. MELARA \\ Purdue University, West Lafayette, Indiana
}

\begin{abstract}
Three experiments examined the domain of visual selective attention (i.e., feature-based selection vs. object-based selection). Experiment 1 extended the requirements of the visual search task by requiring a feature discrimination response to target elements presented for short durations (30-105 msec). Targets were embedded in 47 distractor elements and were defined by either a distinct color or a distinct orientation. Observers made a discrimination response to either the target's color or its orientation. When the target-defining feature and the feature to be discriminated were the same (matched conditions), accuracy was enhanced relative to when these features belonged to separate dimensions (mismatched conditions). In Experiment 2, similar results were found in a task in which the target-defining dimension varied from trial to trial and observers performed both color and orientation discriminations on every trial. The results from these two experiments are consistent with feature-based attentional selection, but not with object-based selection. Experiment 3 extended these findings by showing that the effect is rooted in the overlap between target and distractor values in the stimulus set. The results are discussed in the context of recent models of visual selective attention.
\end{abstract}

The objects that fill our visual world vary in shape, color, size, and use. Successful performance in visual tasks, whether in the real world or in the laboratory, requires that we identify, act upon, or manipulate these objects in some way. Moreover, in all visual tasks, we are called upon to select only some (often one) of the many objects present simultaneously in a scene for further processing. Thus, to operate within a visual environment, cognitive systems must continually accomplish two interrelated feats. The first is to locate the object(s) of current interest within the crowd of irrelevant objects that surround it. The second is to extract information from the selected object(s) - and only the selected one(s) - to guide performance in the task at hand. Systems of human visual cognition typically achieve both these goals superbly, allowing us to interact with our world with little difficulty.

Much research and theorizing have been devoted to understanding how attentional systems select visual objects for processing. Two classes of models have been proposed. According to one class, the object itself is the basic

This research was supported by National Institute of Health Grant NS28617. This article is based in part on a doctoral dissertation submitted by J.R.W. Mounts to Purdue University. We thank Zygmunt Pizlo, Robert Proctor, Richard Schweickert, Howard Whiteman, and Noriko Yamagishi for useful discussions regarding this work, and Art Kramer, Morris Goldsmith, Juan Lupiáñez, and an anonymous reviewer for helpful comments on an earlier version of this manuscript. Correspondence should be addressed to J. R. W. Mounts, Department of Psychology, State University of New York at Geneseo, 1 College Circle, Geneseo, NY 14454 (e-mail: mounts@uno.cc.geneseo.edu). unit through which selection operates (see, e.g., Duncan \& Humphreys, 1989, 1992), whereas, according to the other, the goal of attention is to select the subordinate features of objects (e.g., Logan, 1996; Treisman, 1988).

To illustrate these views, consider an object of a certain color and shape. On the object-based view, the color and shape attributes are fully integrated into a unitary perceptual representation before attention is initiated. Consequently, focus is directed to the unified object, selecting all of its constituent features simultaneously (e.g., both its color and its shape). In other words, having knowledge of the object's color also implies having knowledge of the object's shape.

Feature-based views, on the other hand, hold that attention selects each of an object's features separately. On this view, the time course for processing an object's color may be quite distinct from the time course for processing an object's shape. Thus, knowledge of color may occur in the absence of knowledge of shape.

In the present set of experiments, we contrasted the object-based view of attentional selection with the featurebased view. We created a situation in which a unique feature caused a target element to "pop out" from an array of distractor elements. To gauge the relative accessibility of two of the target's features, we asked observers to identify or discriminate the values of those features. If the objects themselves are the primary unit of attentional selection, then access to one feature of an object implies simultaneous access to its other feature. If attention operates at the level of the object's features, however, then information 
about the different features need not be available at the same time, suggesting that we can create situations in which one of the object's features has a clear selection advantage over the other. To understand these predictions more fully, let us explore the particulars of some representative object-based and feature-based models.

\section{Object-Based Selection}

The view that the currency of attention is the integrated object is a popular one in modern cognitive theorizing (e.g., Driver \& Baylis, 1989; Duncan, 1984; Duncan \& Humphreys, 1989; Kahneman, Treisman, \& Gibbs, 1992). Duncan and Humphreys's $(1989,1992)$ attentional engagement theory (AET) is representative of the view that attention selects perceptual objects. According to AET, preattentive processes combine an object's features (which have been processed to the categorical level) into a structural unit. Attention operates only after the formation of these structural units (perceptual objects), by selecting these objects for entry into visual short-term memory (VSTM). Once an object representation enters VSTM, all aspects of that object become available to inform further cognitive and motor processes, regardless of how or why the object was selected.

Duncan and Humphreys (1989) have stated that the efficiency of selecting a target object for entry into VSTM is determined by the perceptual similarity in the array between the target and the distractors, as well as by the homogeneity among the distractors (see also Duncan, 1989; Nothdurft, 1992, 1993). This tenet helps explain the ease of typical feature search tasks in which the targets pop out from their background; here each target is dissimilar to its distractors, while the distractors are very similar to one another. The similarity among the distractors allows the attentional system to inhibit distractors as an ensemble, thereby facilitating selection of the target object for entry into VSTM.

In the present study, targets were defined by the presence of a unique feature value embedded among a set of relatively homogeneous distractors. In line with objectbased accounts, under these conditions the attentional system should be able to efficiently inhibit all distractor elements, efficiently selecting the target object, pop-out feature, and all other features together.

\section{Feature-Based Selection}

An alternative to models proposing object-based selection is the view that attentional selection is coordinated at the level of an object's features; attention selects an object by selecting the features that constitute the object. On this account, attentional selection is (at least partially) independent for the different features of an object. Two different kinds of models from the visual attention literature are consistent with the view that features are the primary unit of attentional selection: (1) space-based models, which propose that attention selects features occupying a specific spatial region (see, e.g., Logan, 1996; Treisman, 1988, 1993), and (2) hybrid models, which as- sume that attention selects an object by separately selecting features linked to a common object representation (see, e.g., Duncan, 1996).

According to space-based models of attention, attending to a specific region of space leads to the selection of features occupying that location; the selected "object" is the combination of features at that location (see, e.g, Logan, 1996; Treisman, 1988, 1993). Logan's CODE theory of visual attention (CTVA) is representative of the view that attention operates by sampling the features from a specified spatial region. In CTVA, the purpose of attention is to obtain evidence about features of objects in the visual environment, where an object is a region of space defined by preattentive grouping processes (accomplished by a CODE module; see Compton \& Logan, 1993; van Oeffelen \& Vos, 1982). These regions are prioritized for order of selection (e.g., through a salience map; see Cave \& Wolfe, 1990; Koch \& Ullman, 1985). Once a region is selected, attention samples features from that location and uses these features as input into a categorization mechanism based on Bundesen's (1990) theory of visual attention (TVA). In CTVA, the operation and efficiency of categorization depends on the nature of the featural input (e.g., similarity relations) and the demands of the visual task. Moreover, the TVA module processes each feature independently. Thus, if the task demands a categorization response on two of an object's features (e.g., its color and its shape), the module will reach its category decisions independently for each dimension. As a result, the processing of each of an object's separate features may follow different time courses. In the present study, we exploit this idea by examining whether we can manipulate the relative rate of processing of different features of an object.

The second kind of model consistent with feature-based selection is actually a hybrid of object- and feature-based models. In describing their object-based AET, Duncan and Humphreys (1989) proposed that processes such as distractor inhibition and target selection operate at the level of fully integrated objects. If we assume instead, as Duncan (1996) does in his integrated competition hypothesis, that distractor inhibition (and target selection) operates at the level of individual feature maps, then attentional selection is feature based. However, models such as Duncan's (1996) are also termed object based (as opposed to space based) because the features are presumed to be linked to an object token (see, e.g., Goldsmith, 1998; Kanwisher \& Driver, 1992). It is this shared link to the object token (as opposed to a shared spatial location) that coordinates the selection of an object's features. Numerous models with a hybrid architecture of this type have been introduced recently (e.g., Duncan, 1996; Houghton \& Tipper, 1994; Schneider, 1995).

Although the mechanisms of selection differ between the two types of feature-based models, both the spacebased models and the hybrid models make identical predictions across our entire set of experiments. For the sake of brevity and clarity, predictions regarding feature-based selection will be couched in terms of space-based models 
(see, e.g., Logan, 1996). However, in the General Discussion, we will return to models such as Duncan's (1996) integrated competition hypothesis to evaluate their ability to handle our specific pattern of results.

\section{The Present Study}

The purpose of the present study was to investigate observers' performance in a modified version of the feature search task as a way of evaluating opposing predictions of object- and feature-based models of attentional selection. The visual search task has been used extensively to understand the processes underlying attentional selection. Unfortunately, many differences between the models - such as those having to do with the availability of object features - are not tapped by traditional search paradigms: Both object-based models (see, e.g., Duncan \& Humphreys, 1989) and feature-based models (e.g., Treisman, 1988) can explain the basic findings from the visual search task, such as the relative efficiencies of feature and conjunction searches. The difficulty in discriminating models can be traced to the dual role attention serves in everyday life; attention is needed to (1) locate relevant objects and (2) extract information from these objects. Most studies employing search tasks have emphasized only the first of these functions because, in these studies, observers are asked merely to determine an object's presence or absence. Hence, the second function of attention is sidestepped, as observers are never asked to make detailed discriminations regarding an object's properties. In the present study, we modified the task by requiring observers to make identifications or discriminations about a specific feature of the target element. This change allowed us to tease apart object-based selection from feature-based selection.

The key to differentiating the models lies in limiting the display duration of feature search. According to both types of models, the detection and localization of targets defined by unique features occurs rapidly. Nonetheless, the attentional mechanisms are not instantaneous; they require time to accomplish their task. Presenting visual displays for a brief duration will tax the limits of either object- or feature-based mechanisms, causing performance to suffer. However, the two types of models differ in their explanation of how and why performance suffers.

According to object-based accounts, limiting the display duration can cause a failure to select the target object (see, e.g., Bundesen, 1990; Duncan \& Humphreys, 1989). In AET, identifying an object's features first requires that attention select that object (an object must enter VSTM to inform further processes). Thus, on trials in which there is a failure to select the target object, we would expect to observe chance accuracy in identifying each and every feature of the target. The object-based view holds that similarity between features of the target and the distractors will influence the ease with which a target can be selected; however, the effects of featural similarity should have no effect on processing beyond target selection. In other words, once an object is selected, there is no reason to expect that differences in a distractor's similarity to a target will differentially affect success at discriminating the target's features.

In feature-based accounts, brief display durations may cause attention to sample featural information from irrelevant spatial locations, in particular features from adjacent distractors. This prediction applies to a variety of metaphors that have been offered for spatial attention, many of which assume an adjustable or fuzzy boundary to the attentional focus (e.g., the zoom lens of C. W. Eriksen \& St. James, 1986; or the gradient filter of LaBerge \& Brown, 1989). Logan (1996) assumed that it is the spatial extent of features that is fuzzy, rather than the spatial focus of attention. In either case, the result is a sampling of irrelevant information from distractors. The errant sampling of distractor features would naturally add noise to the perceptual information on which decisions are made, thereby disrupting observers' ability to make accurate feature identifications. The disruptive effects of noise are likely to be intensified when features of the distractor are perceptually similai to those of the target, and as a result cannot be rejected as irrelevant to the discrimination process.

In sum, if inadequate time is available for attentional mechanisms to operate-that is, if performance is kept below asymptote for a given target discrimination - both feature- and object-based models predict that the ability to extract information accurately from such a target will be compromised. However, the two types of model differ in explaining how performance suffers. Object-based models hold that limiting the exposure duration should affect selection of the target as a whole and should therefore impact all features of the object equally. Feature-based models, on the other hand, predict that limiting exposure duration may add noise differentially to the identification of the separate features of an object. Experiment 1 was designed to evaluate these predictions.

\section{EXPERIMENT 1}

In each experiment of the present study, an array of simple stimuli (lines that varied in orientation and color) was displayed for a brief $(30-105 \mathrm{msec})$ duration. One item in the display (the target) was defined by a distinct orientation or color value. The dimension containing the distinct value (i.e., orientation or color) was called the pop-out dimension. Figure 1 depicts a color pop-out display, and Figure 2 depicts an orientation pop-out display. Once the observer identified the target via the pop-out dimension, he/she was asked to decide (discriminate) between two possible values of the target along a dimension. This discrimination dimension was either color or orientation; it may or may not have been the same dimension as the pop-out dimension. Across conditions, the pop-out dimension was combined factorially with the discrimination dimension. Thus, in half the conditions, the popout and discrimination dimensions matched, whereas in the other half, they mismatched. 


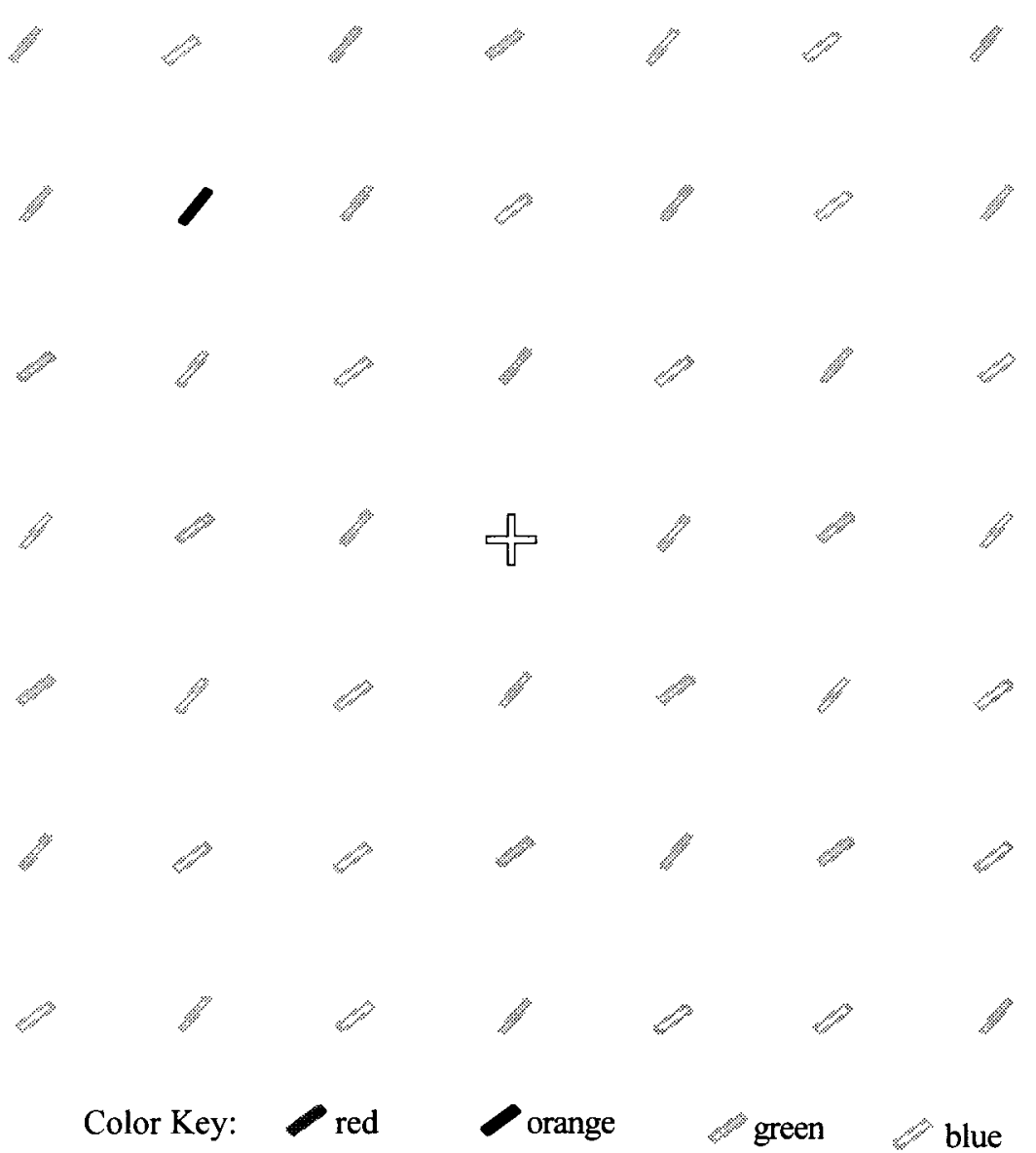

Figure 1. An example of a color pop-out stimulus display.

When the pop-out and discrimination dimensions corresponded (matched conditions), the values of the distractors on the discrimination dimension (e.g., color) were perceptually distinct (e.g., in color) from the potential values of the target on that dimension; that is, in the matched conditions, there was no overlap between values from the target set and the values from the distractor set. This had two effects: (1) The target popped out on the discrimination dimension, and (2) the distractor values were relatively unconfusable with the potential target values. When the discrimination dimension and pop-out dimensions differed (mismatched conditions), however, the individual distractor items took on one of the two target values on the discrimination dimension; that is, in the mismatched conditions, there was complete overlap between values from the target set and values from the distractor set. This had two effects, which were opposite from those in the matched conditions: (1) The target element did not pop out on the discrimination dimension-in fact, it was impossible for the observer to determine which element was the target solely on this dimension; (2) the distractors' values on the discrimination dimension were potential target values.
According to object-based models of attentional selection, attention operates after the features of an object have been conjoined, selecting all aspects of an object. The efficiency of this selection is controlled in part by the similarity between the target and the distractors, and in part by the homogeneity of the distractors. Consequently, the efficiency of target selection is determined by the characteristics of the stimulus and should be independent of the actual discrimination being made with respect to the target. For example, if a target is defined by a unique color, selection ease should not depend on whether observers are asked to make orientation or color discriminations of the target; according to object-based models, the same process of selecting the target object to control the response would be required to complete either type of task. In the same vein, any failure to select the target should impact color and orientation identifications equally, since the information required to complete either type of task would be absent (e.g., from VSTM, according to AET).

In mapping object-based explanations to the present task, we would expect specific patterns of results in an analysis of variance (ANOVA) of feature discrimination 


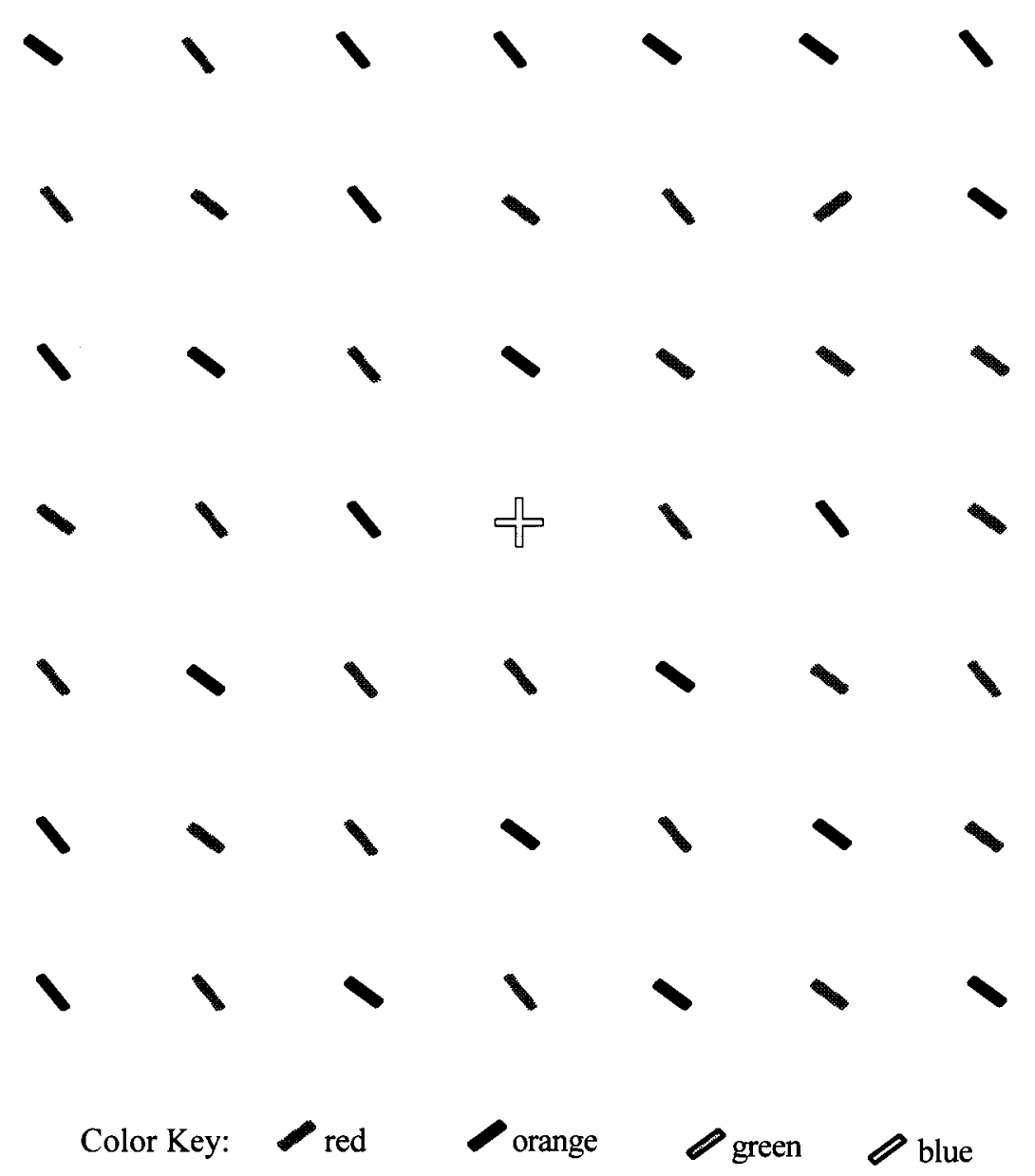

Figure 2. An example of an orientation pop-out stimulus display.

performance. First, it is possible that we might observe a main effect of pop-out dimension-for example, better performance to color pop-outs than to orientation popouts-since the similarity between target and distractor values is presumed to influence the selection process. In other words, color pop-outs may be more salient than orientation pop-outs (or vice versa). Similarly, a main effect of discrimination dimension might be observed-indicating, for example, that orientation discrimination is easier than color discrimination-since the discriminability of the target values can influence the perceptual discrimination process. In other words, for a given observer, the two orientation targets may be more perceptually distinct than are the two color targets (or vice versa). The critical result, however, involves the interaction between the pop-out dimension and the discrimination dimension. Because all aspects of an object become available when it is selected, it should not matter whether the pop-out dimension is the same as (matched condition) or different from (mismatched condition) the discrimination dimension. Hence, according to object-based accounts, at preasymptotic display durations, one would expect no interaction between the pop-out dimension and the dis- crimination dimension in performance accuracy on our modified search task.

Feature-based models of attention predict a different pattern of performance. A failure of attention is explained as the erroneous sampling of features from distractor elements along with the relevant features of the target. According to this view, the "noise" introduced by the irrelevant (distractor) samples will hamper discrimination performance. More specifically, feature-based models predict that the degree of disruption will differ by condition in our modified feature search task: In the mismatched conditions, the irrelevant distractor values are potential target values, and so will introduce more decision noise, thereby detracting more than the distractor values in the matched conditions, where the distractors are perceptually distinct from the targets on the discrimination dimension. Feature-based models thus predict a preasymptotic performance advantage for matched conditions over mismatched conditions. Hence, in addition to possible main effects of pop-out dimension and discrimination dimension, feature-based models make the clear prediction of a statistical interaction between pop-out dimension and discrimination dimension. This prediction 
is at odds with the prediction from object-based accounts, thereby providing an unambiguous route for distinguishing the two views.

Method
Observers
Four participants, including J.M., served as observers. The 3
other observers were recruited from the Purdue University com-
munity and were paid \$6 per hour for their participation. All ob-
servers had normal or corrected-to-normal vision and were screened
for color blindness using pseudo-isochromatic color plates. Three
of the observers (J.M., A.W.M., and A.K.) had extensive experience
as observers in psychophysical experiments.

\section{Apparatus}

All experiments were controlled by a Macintosh LC III. Trial initiation and responses were made via the computer keyboard. Stimuli were presented on a 14-in. Macintosh RGB color monitor. The observers viewed the monitor from a distance of $50 \mathrm{~cm}$, with head stabilization aided through the use of a chin and forehead rest. Experiments were conducted in a darkened room.

\section{Stimuli and Tasks}

On each trial, a $7 \times 7$ array of colored line segments (minus the center element, which remained a fixation cross) appeared on a dark gray background (luminance: $1.15 \mathrm{~cd} / \mathrm{m}^{2}$ ). Each line segment subtended a vertical visual angle of $.628^{\circ}$ and a horizontal visual angle of $.089^{\circ}$. The spacing between the centers of adjacent line segments was $2.51^{\circ}$ square, and the entire display subtended a visual angle of $15.32^{\circ}$ square.

The observer's task was to identify either the color or the orientation of the target. The target was physically very different from the other elements in the display (distractors) in either color or orientation (the pop-out dimension); the large physical difference had the effect of causing the target to pop out from the distractors. The target could appear in one of four possible locations within the $7 \times 7$ array, each location equidistant $\left(7.07^{\circ}\right)$ from a central fixation point: the upper left quadrant (second column, second row), upper right quadrant (sixth column, second row), lower left quadrant (sixth column, second row), or the lower right quadrant (sixth column, sixth row).

Each of the four possible combinations of pop-out dimension and discrimination dimension was tested: (Condition 1) color discrimination of targets that popped out on color; (Condition 2) orientation discrimination of targets that popped out on color; (Condition 3) orientation discrimination of targets that popped out on orientation; (Condition 4) color discrimination of targets that popped out on orientation. Notice that in two of the conditions, the pop-out and discrimination dimensions matched (Conditions 1 and 3 ), and in two conditions, they mismatched (Conditions 2 and 4). Targets that popped out on color were either orange (CIE coordinates: $x=.51, y=.42)$ or red $(x=.61, y=.35)$; distractors were either green $(x=.25, y=.48)$ or blue $(x=.21, y=.29)$. Targets that popped out on orientation were either $38^{\circ}$ or $52^{\circ}$ clockwise relative to vertical; distractors were either $308^{\circ}$ or $322^{\circ}$. In all displays, luminance $\left(\approx 4.90 \mathrm{~cd} / \mathrm{m}^{2}\right)$ was equated individually for each observer through the use of heterochromatic flicker photometry.

Each display appeared for $30,45,60,75,90$, or $105 \mathrm{msec}$. Each stimulus element in the display was individually masked immediately after stimulus presentation. Each mask was an ensemble of green $\left(x=.28, y=.60\right.$, luminance: $\left.10.29 \mathrm{~cd} / \mathrm{m}^{2}\right)$ vertical and horizontal line segments, and red $\left(x=.66, y=.30\right.$, luminance: $\left.14.11 \mathrm{~cd} / \mathrm{m}^{2}\right)$ $45^{\circ}$ and $315^{\circ}$ diagonals, equal in size to the preceding stimulus elements. The masking display was response terminated.

The four conditions are described in detail below. Conditions 1 and 3 were matched conditions (i.e., the target popped out on the discrimination dimension), and Conditions 2 and 4 were mismatched conditions (i.e., the target did not pop out on the discrimination dimension). Note that the stimulus display in each of the two matched conditions had a corresponding and identical stimulus display in the mismatched conditions.

Condition 1: Color pop-out/color discrimination. Half of the distractors were blue, and half green. Half were oriented at $38^{\circ}$, and half at $52^{\circ}$. The target was also either red or orange and had an orientation of either $38^{\circ}$ or $52^{\circ}$. The observers' task was to identify the color of the target. A sample display appears in Figure 1. As one can see, the target could be found in the display by its color, but not its orientation. In this example, the correct response is "orange."

Condition 2: Color pop-out/orientation discrimination. The stimulus displays were identical to those used in Condition 1 (Figure 1). In Condition 2, the observer's task was to identify the orientation of the target, either $38^{\circ}$ or $52^{\circ}$. Again, the target could be found in the display by its color, but not its orientation. In the example in Figure 1, the correct response in this condition is " $38^{\circ}$."

Condition 3: Orientation pop-out/orientation discrimination. Half of the distractors were oriented at $308^{\circ}$, and half at $322^{\circ}$. Half were orange and half red. Targets were also either red or orange, but were oriented at $38^{\circ}$ or $52^{\circ}$. The target thus popped out on orientation, but not color. The observer's task was to identify the orientation of the target. An example of this type of display appears in Figure 2. Here, the correct response is " $52^{\circ}$."

Condition 4: Orientation pop-out/color discrimination. The stimulus displays were identical to those used in Condition 3 (Figure 2) except that in Condition 4, the observer's task was to identify the color value of the target. In the example in Figure 2, the correct response is "red."

\section{Procedure}

To aid in making comparisons across experiments, observers completed Experiments 1 and 2 in counterbalanced order, with each observer completing all sessions of one experiment before proceeding to the next. Observers J.M. and F.H. completed Experiment 1 first, whereas Observers A.W.M. and A.K. completed Experiment 2 first.

Experiment 1 included five sessions; the first session served as practice, with the data excluded from the analyses. All four conditions were performed in counterbalanced order within each session. Within each condition, observers completed six blocks of 96 trials, with each block using a unique target-distractor display duration $(30,45,60,75,90$, or $105 \mathrm{msec})$, presented in random order. Before beginning each condition, observers completed a warm-up block of 24 trials at a display duration of $75 \mathrm{msec}$. Each session lasted approximately $1 \mathrm{~h}$.

Each trial began when a gray fixation cross (luminance: $5.78 \mathrm{~cd} / \mathrm{m}^{2}$ ) appeared in the center of the screen. The observer fixated the cross and initiated stimulus presentation by pressing a key on the keyboard. The stimulus display then appeared for the display duration designated for that block of trials, followed immediately by the masking display. Auditory error feedback (a tone) followed incorrect responses.

\section{Results}

Accuracy data (proportions correct) were examined in each condition at each display duration for each observer to evaluate whether performance differed for matched versus mismatched conditions. The results appear in the four panels of Figure 3 (color discriminations for each of the 4 observers) and Figure 4 (orientation discriminations).

The accuracy data were analyzed using a randomized block design with repeated measures (Winer, 1971), treating observer (four levels) as a random factor and 

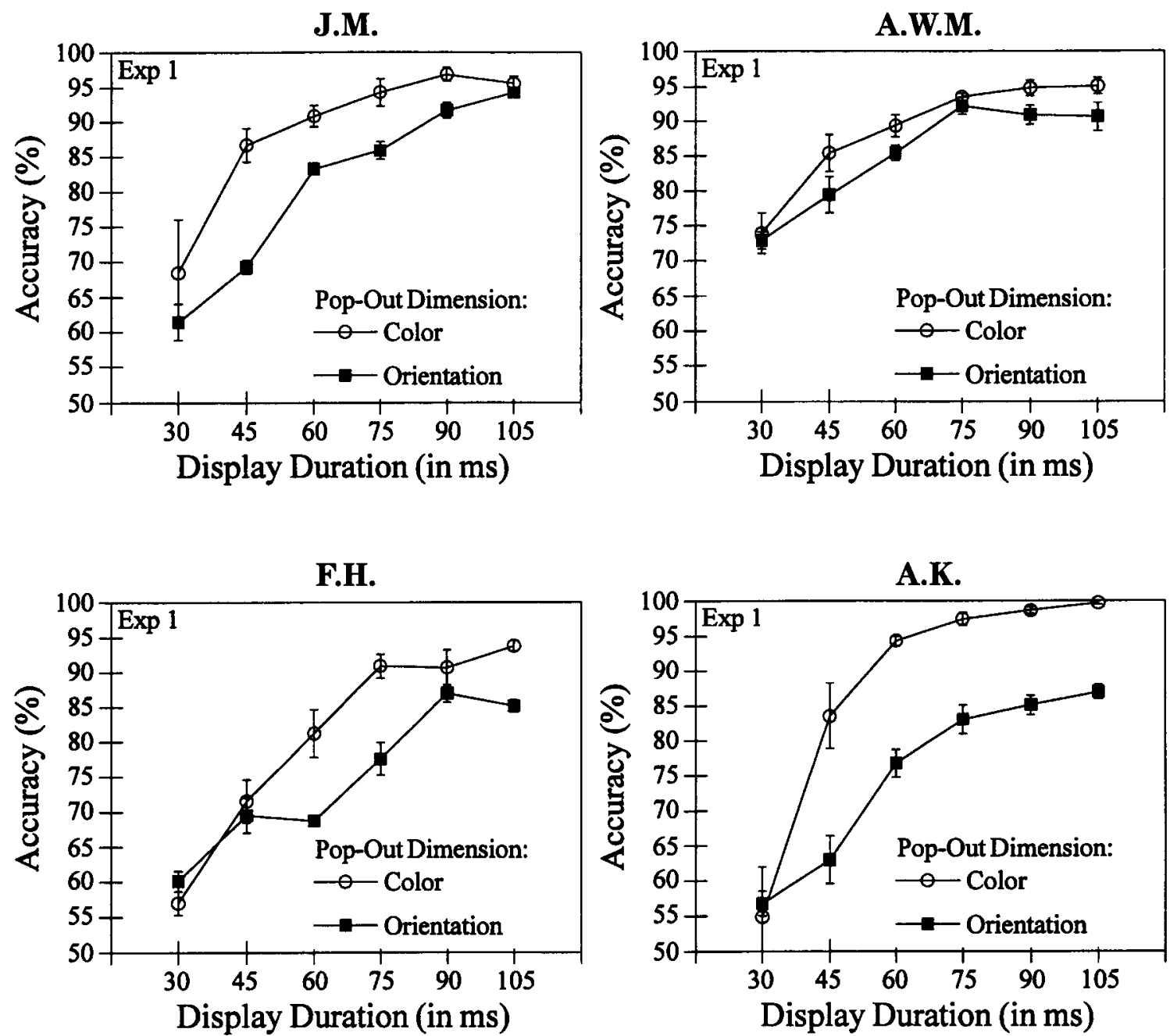

Figure 3. Color discrimination accuracy from Experiment 1 for each of the 4 observers as a function of display duration and pop-out dimension. Error bars depict $\pm 1 S E$. Open symbols and filled symbols correspond to matched and mismatched conditions, respectively.

discrimination dimension (two levels), pop-out dimension (two levels), and display duration (six levels) as fixed factors, with session providing the error term for each cell. Reliable main effects were obtained for observer $\left[F(3,12)=164.83, M S_{\mathrm{e}}=.0011, p \leq .001\right]$ and display duration $\left[F(5,15)=64.11, M S_{\mathrm{e}}=.0108, p \leq .001\right]$. There was also a significant three-way interaction among discrimination dimension, pop-out dimension, and display duration $\left[F(5,15)=14.65, M S_{\mathrm{e}}=.0023, p \leq .001\right]$. The observer factor interacted with each of the other three factors: discrimination dimension $[F(3,12)=10.34$, $\left.M S_{\mathrm{e}}=.0046, p \leq .01\right]$, pop-out dimension $[F(3,12)=$ $\left.7.35, M S_{\mathrm{e}}=.0035, p \leq .005\right]$, and display duration $\left[F(15,60)=5.37, M S_{\mathrm{e}}=.0020, p \leq .001\right]$. Further, a three-way interaction appeared among observer, discrimination dimension, and pop-out dimension $[F(3,12)=$ $\left.8.27, M S_{\mathrm{e}}=.0017, p \leq .005\right]$. Most important, the twoway interaction between discrimination dimension and pop-out dimension was reliable $\left[F(1,3)=26.21, M S_{\mathrm{e}}=\right.$ $.0144, p \leq .01]$, indicating a matched advantage.

\section{Discussion}

Examining Figures 3 and 4, one can see clearly that each observer evinced a matched advantage during all pre-asymptotic display durations. That is, across the two judgment types, observers were more accurate in their discriminations when the discriminations were made to values on the pop-out dimension (i.e., the target and distractor values had no overlap) than when the discriminations were made to values on the non-pop-out dimension (i.e., the distractor values overlapped completely with the target values).

Further examination of Figures 3 and 4 reveals that the level of asymptotic performance-as well as the rate at which this performance limit was reached--differed for the two pop-out dimensions and for each of the 4 ob- 

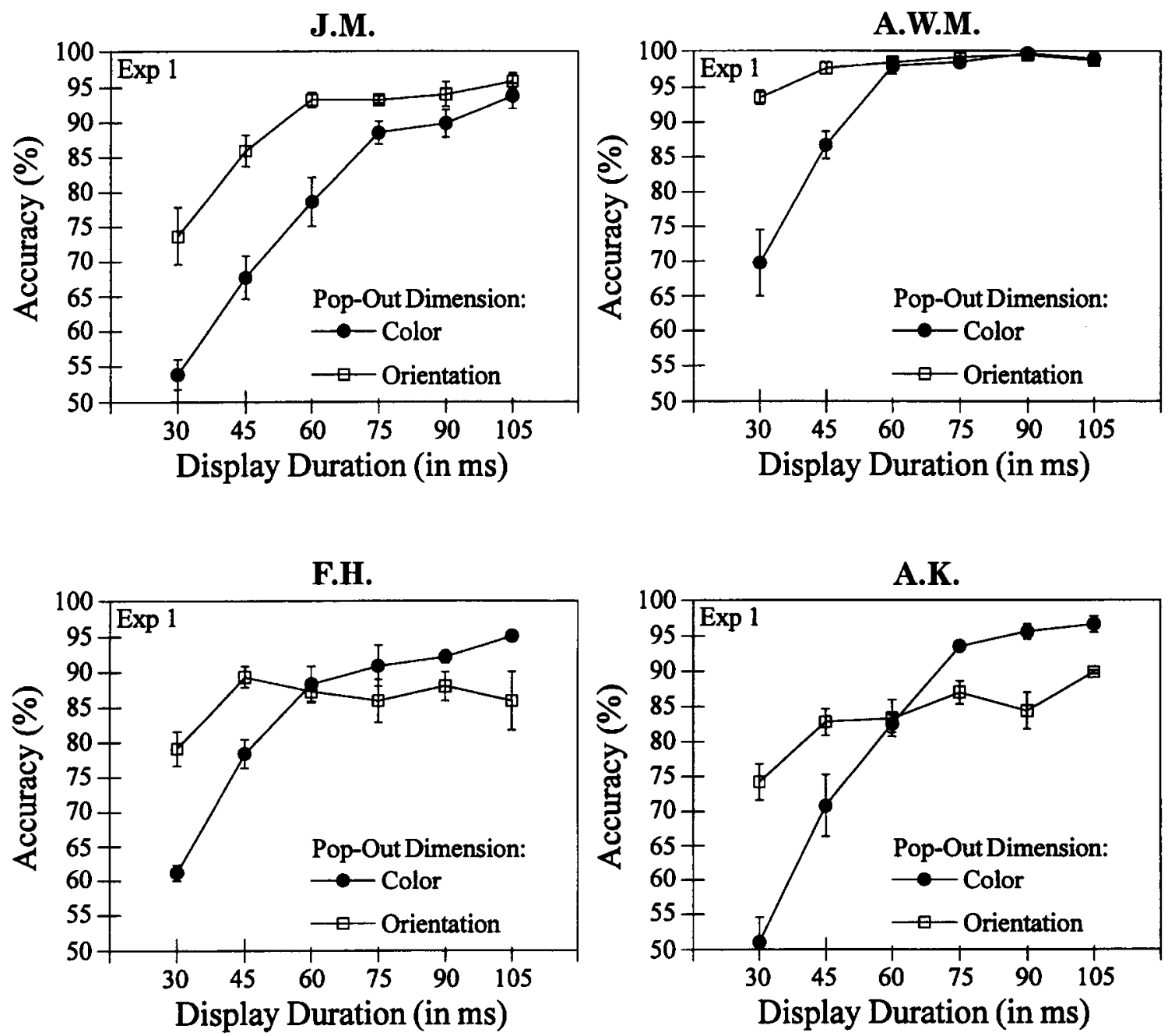

\begin{abstract}
Figure 4. Orientation discrimination accuracy from Experiment 1 for each of the 4 observers as a function of display duration and pop-out dimension. Error bars depict $\pm 1 S E$. Open symbols and filled symbols correspond to matched and mismatched conditions, respectively.
\end{abstract}

servers. For example, in Observer A.K.'s data, color popouts resulted in higher asymptotic levels of performance than did orientation pop-outs. This difference in the asymptotes of performance is probably the result of the relative salience of the two types of pop-out for the individual observers. The differences in asymptotic performance by observer and pop-out dimension underscore the importance of examining performance over a range of display durations, allowing us to disentangle effects due to attentional selection mechanisms from other factors controlling performance. As noted, regardless of these differences in absolute levels of asymptotic performance, prior to asymptote all 4 observers showed a matched advantage for both color and orientation discriminations.

The results of Experiment 1 are consistent with the predictions offered by feature-based accounts of visual attention, but appear to be inconsistent with the predictions of object-based models. All 4 observers experienced a matched advantage: Their identification performance was better in the matched conditions (i.e., Conditions 1 and 3) than in the mismatched conditions (i.e., Conditions 2 and 4), regardless of the dimension on which the discrimination was being made.

Feature-based models of attention predicted the results of Experiment 1 . In this view, it is assumed that attention may blend samples of information from distractor locations with samples of information from the target location, thereby disrupting the discrimination process. At short exposure durations, the low signal-to-noise ratio created by the blended samples should hamper discrimination performance. Moreover, the degree of disruption to the discriminations should intensify with the similarity between distractor and target values. In the mismatched conditions, the similarity between targets and distractors was maximal because distractor values were split equally among the two possible target values along the discrimi- 
nation dimension. For example, in the orientation pop-out/ color discrimination condition, a mismatched condition, the target was either red or orange, while each of the distractors was either red or orange. Thus, it would be impossible to differentiate between the distractors and the target along the discrimination dimension. In the matched conditions, distractor values differed from the potential target values. For example, in the color pop-out/color discrimination condition, a matched condition, the target was either red or orange, while the distractors were either blue or green. A feature-based theory such as Logan's (1996) CTVA predicts relatively less interference (higher accuracy) in the matched conditions because the task irrelevance of the distracting features allows them to be filtered at the decision stage. Such filtering based on feature values is impossible in the mismatched conditions. Thus, the theory is able to explain the observed interaction between the pop-out dimension and the discrimination dimension, expressed here as the matched advantage.

The matched advantage is more difficult to reconcile with object-based selection. According to this class of models, two factors might have influenced performance in our task: (1) the ease of target selection and (2) the perceptual discriminability of target values. Yet neither of these factors is able to account for the statistical interaction obtained between the pop-out dimension and the discrimination dimension. The ease with which targets could be selected - defined here as the distinctiveness of the targets relative to the distinctiveness of the distractors-was purposely equated across conditions. This was achieved by utilizing the same type of pop-out (e.g., orientation popouts) across corresponding matched and mismatched conditions. For example, the displays for the matched orientation pop-out/orientation discrimination conditions were identical to the displays for the mismatched orientation pop-out/color discrimination conditions; the ease of selecting the pop-out target was thus equated for these two tasks. The perceptual discriminability of the target values were also equated across matched and mismatched conditions for a specific type of identification. This was achieved by making the identifications performed in the matched conditions exactly the same as the identifications performed in the mismatched conditions. For example, in the matched orientation pop-out/orientation discrimination condition, observers were asked to discriminate between target values of $38^{\circ}$ and $52^{\circ}$, the same target values they were asked to discriminate in the mismatched color pop-out/orientation discrimination condition. Thus, although object-based models can account for the main effects of pop-out dimension (due to to target-distractor distinctiveness) and discrimination dimension (due to discriminability) seen for individual observers, it appears, at least at first glance, that these models are unable to account for the interaction between the pop-out dimension and the discrimination dimension that produced the matched advantage.

Closer inspection of the task demands in Experiment 1, however, reveals a possible mechanism by which an object-based attentional model might produce a matched advantage. In many object-based accounts, the rate at which objects are selected is determined in part by how relevant the features of those objects are to the task at hand (e.g., Bundesen, 1990; Duncan \& Humphreys, 1989, 1992). For example, in AET, Duncan and Humphreys (1989) assigned attentional weights to the target features to modulate the speed at which objects possessing those features were selected. In the matched conditions, the observers searched for a target defined by the same dimension on which they were to make a discrimination. For example, in the matched color pop-out/color discrimination condition, observers were preparing to make a red/orange discrimination, while the target was defined by possession of one of these two values. This was not true in the mismatched conditions, in which the features defining the target and the features to be discriminated were drawn from different dimensions. For example, in the mismatched orientation pop-out/color discrimination task, the target was defined by an orientation of either $38^{\circ}$ or $52^{\circ}$, but observers made a red/orange discrimination. It is possible that preparing to make a specific discrimination (e.g., red/orange) boosted the attentional weight on those features, therefore speeding their selection. On this interpretation, the matched advantage arises because the features driving selection are also the features being discriminated. Thus, it is possible that the task demands influenced the speed with which targets were selected, with targets being selected more quickly in the matched conditions. This object-based account of the matched advantage was tested in Experiment 2.

\section{EXPERIMENT 2}

Two viable explanations have been offered for the matched advantage. Our interpretation of object-based models emphasizes the task demands; the matched conditions yield a performance advantage because the search required for the target and the discrimination involves the same stimulus dimension. Feature-based models, on the other hand, concentrate on stimulus characteristics in the two types of tasks - specifically, on the distractortarget relationships. In this view, the inability to differentiate target from distractor values on the discrimination dimension disrupts performance in the mismatched tasks. These two explanations can be distinguished empirically: By changing the task slightly, the task level dependencies between the pop-out dimension and the discrimination dimension can be removed, while maintaining the stimulus relations at the trial level.

The tasks used in Experiment 2 were similar to those used in Experiment 1 except that the pop-out dimension (i.e., how the target was defined) was now mixed rather than blocked, and observers performed absolute identification (i.e., identification of both the color and the orientation value), rather than identification along a single dimension. These modifications removed any associations between the pop-out dimension and the discrimination dimension: Observers had to prepare to locate a target that could have been defined by either dimension, and they 
had to make a discrimination on both sets of features (i.e., both orientation and color). As a result, the attentional weights for both sets of features should be equally high. Nonetheless, matched and mismatched conditions can still be distinguished at the trial level. On any given trial, the target popped out on either the color dimension or the orientation dimension. On a trial-to-trial basis, we assessed matched performance through discriminations made to the dimension on which the target popped out; mismatched performance was assessed by discriminations of the dimension that was not the pop-out dimension. In this situation, object-based models predict that the matched advantage should disappear in Experiment 2the task-level contingencies present in Experiment 1 have been removed. According to the feature-based account, however, the task changes should not alter the matched advantage observed in Experiment 1 since the trial-level relationships between the target and distractor values are still in place.

\section{Method}

\section{Observers, Apparatus, and Stimulus Values}

The observers and apparatus were identical to those of Experiment 1 . Trial initiation, presentation, and poststimulus masking occurred as in the previous experiment. The same two target values and distractor values for color and orientation as those from Experiment 1 were used.

\section{Conditions, Trial Types, and Procedure}

The target stimuli were created by combining the two target values for color with the two target values for orientation, yielding four possible targets: orange $/ 38^{\circ}$, orange $/ 52^{\circ}$, red $/ 38^{\circ}$, and red $/ 52^{\circ}$. The observers were asked to make both an orientation and a color discrimination of the target item on each trial. This was accomplished by responding with one of four keys (i.e., the 1,2,4, and 5 keys on the number pad), with each response key corresponding to one of the four possible target stimuli. The stimulus displays themselves were identical to those used in Experiment 1; the target item was designated by a distinct color or orientation value. In this experiment, however, the dimension defining the target varied randomly from trial to trial. For example, color pop-outs occurred when one of the four possible targets appeared among blue and green distractors, oriented at $38^{\circ}$ and $52^{\circ}$; orientation pop-outs occurred when one of the four targets appeared among orange and red distractors, oriented at $308^{\circ}$ and $322^{\circ}$. Matched and mismatched discriminations were determined on a trial-by-trial basis. On trials in which the target's color popped out, color discriminations defined the matched conditions, and orientation discriminations defined the mismatched conditions. On trials in which the target's orientation popped out, orientation discriminations defined the matched conditions, and color discriminations defined the mismatched conditions. A tone followed incorrect responses, with visual feedback designating the dimension(s) on which the error was made.

Each experimental session consisted of six blocks of 192 trials. The display duration was manipulated between blocks of trials, as in Experiment 1. The order of appearance of the display duration within each experimental session was randomized. Each observer completed one practice session and four experimental sessions, with each session lasting approximately $45 \mathrm{~min}$.

\section{Results}

Color and orientation discriminations were partitioned according to the pop-out dimension, permitting us to as- sess performance in each of four types of conditions: color matched, color mismatched, orientation matched, and orientation mismatched. As in Experiment 1, the proportions correct were examined in each condition at each display duration. The four panels of Figure 5 show the accuracy of color discriminations in matched and mismatched conditions for each of the 4 observers. The four panels of Figure 6 contain the same information for the matched and mismatched orientation discriminations.

The accuracy data were analyzed as in Experiment 1, using a randomized block design with repeated measures, treating observer (four levels) as a random factor and discrimination dimension (two levels), pop-out dimension (two levels), and display duration (six levels) as fixed factors, with session providing the error term for each cell. Reliable main effects were found for both observer $\left[F(3,12)=24.63, M S_{\mathrm{e}}=.0092, p \leq .001\right]$ and display duration $\left[F(5,15)=37.00, M S_{\mathrm{e}}=.0130, p \leq .001\right]$. The observer factor interacted with the other three factorsobserver and discrimination dimension $[F(3,12)=12.03$, $\left.M S_{\mathrm{e}}=.0060, p \leq .001\right]$, observer and pop-out dimension $\left[F(3,12)=5.76, M S_{\mathrm{e}}=.0059, p \leq .05\right]$, and observer and display duration $\left[F(15,60)=7.94, M S_{\mathrm{e}}=.0016, p \leq\right.$ $.001]$. Also, there was a three-way interaction between observer, discrimination dimension, and pop-out dimension $\left[F(3,12)=5.05, M S_{\mathrm{e}}=.0028, p \leq .05\right]$ and a fourway interaction involving all of the factors $[F(15,60)=$ $\left.1.99, M S_{\mathrm{e}}=.0016, p \leq .05\right]$. Most important, the two-way interaction between discrimination dimension and popout dimension was reliable $\left[F(1,3)=11.19, M S_{\mathrm{e}}=.0142\right.$, $p \leq .05]$. An examination of Figures 5 and 6 reveals that this interaction takes the form of the matched advantage, as in Experiment 1.

The pattern of results depicted in Figures 5 and 6 bears a striking resemblance to the data of Experiment 1 (cf. Figures 3 and 4). In order to evaluate the apparent similarity of the results from Experiments 1 and 2 quantitatively, statistical analyses were performed. Figures 3-6 show clearly that the pattern of results for each individual observer was very consistent from Experiment 1 to Experiment 2. Nonetheless, each observer reached his/her performance asymptote at a unique display duration, and the absolute asymptotic levels differed across observers and across discrimination dimensions. Because of these intersubject differences, and because of the increased power afforded by combining the data from two experiments, our interexperiment comparisons were performed for each observer separately. Recall that the order of completion of these two experiments was counterbalanced across observers, with J.M. and F.H. performing Experiment 1 first, and A.W.M. and A.K. performing Experiment 2 first.

\section{Comparison of Experiments 1 and 2}

The data for each observer from Experiments 1 and 2 were subjected to separate ANOVAs, with the withinsubjects factors of experiment (two levels), discrimination dimension (two levels), pop-out dimension (two levels), 

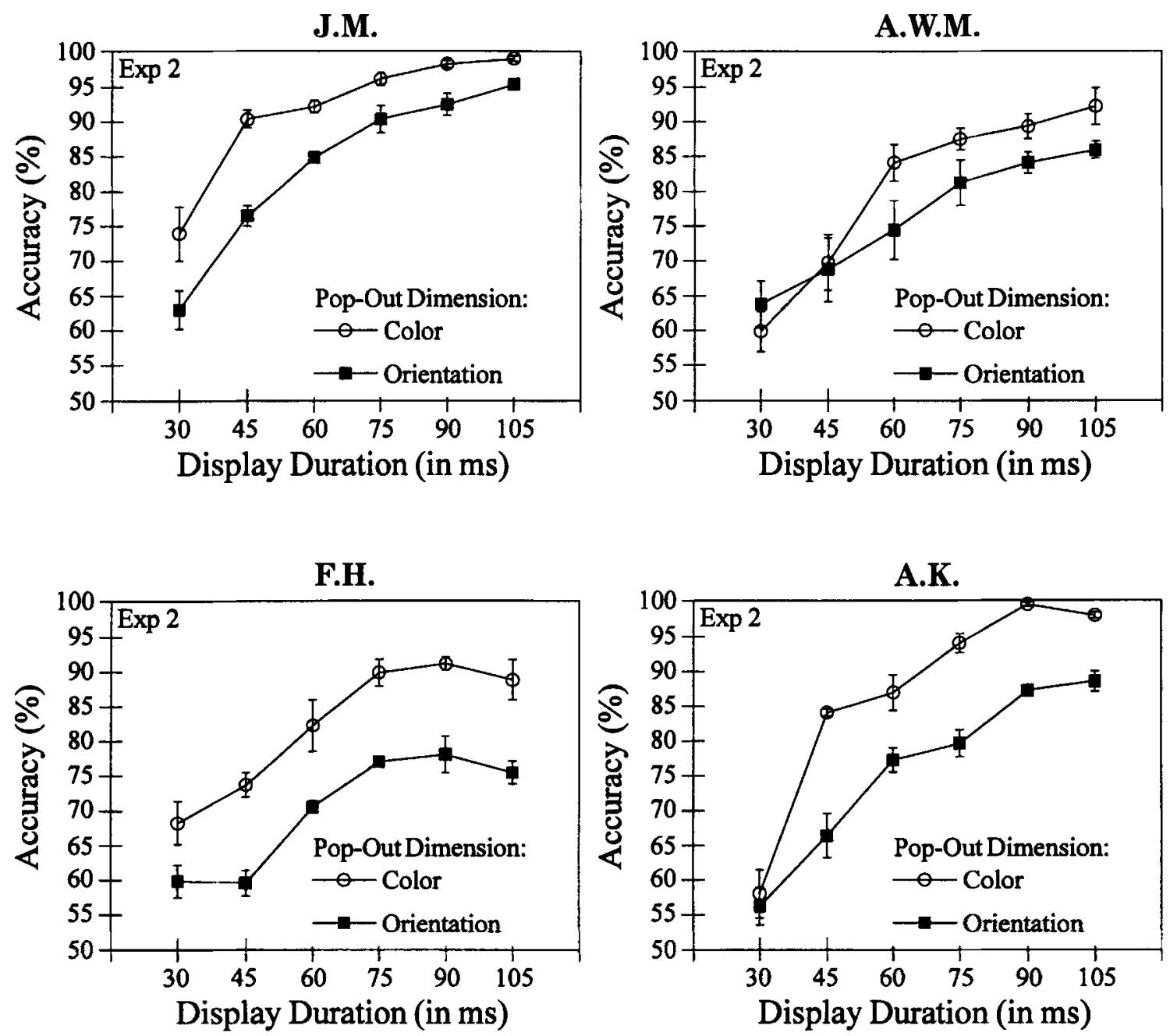

Figure 5. Color discrimination accuracy from Experiment 2 for each of the 4 observers as a function of display duration and pop-out dimension.

and display duration (six levels). We were interested in the main effect of experiment, and in whether experiment would interact with any of the other factors. We were especially interested in the three-way interaction between experiment, discrimination dimension, and pop-out dimension, since the matched advantage resulted in an interaction between pop-out and discrimination dimensions. Observer J.M.'s data yielded a main effect of experiment $\left[F(1,6)=21.71, M S_{\mathrm{e}}=.0018, p \leq .005\right]$, with Experiment 2 yielding more accurate performance. However, the experiment factor did not yield any reliable interactions. Similar results were obtained for Observer A.W.M., who displayed a main effect of experiment $[F(1,6)=8.88$, $\left.M S_{\mathrm{e}}=.0320, p \leq .05\right]$; in this case, an accuracy advantage was observed for Experiment 1. Again, as for J.M., no significant interactions involving experiment were found. Neither the main effect of experiment nor interactions involving experiment approached significance for Observer F.H. or A.K.
Interactions among pop-out dimension and discrimination dimensions (signifying the matched advantage) were reliable for all 4 observers: J.M. $[F(1,6)=283.57$, $\left.M S_{\mathrm{e}}=.0015, p \leq .001\right]$, A.W.M. $\left[F(1,6)=103.49, M S_{\mathrm{e}}=\right.$ $.0016, p \leq .001]$, F.H. $\left[F(1,6)=31.69, M S_{\mathrm{e}}=.0030, p \leq\right.$ $.005]$, and A.K. $\left[F(1,6)=73.38, M S_{\mathrm{e}}=.0026, p \leq .001\right]$. Three-way interactions involving display duration, popout dimension, and discrimination dimension were also reliable for all 4 observers: J.M. $\left[F(5,30)=14.00, M S_{\mathrm{e}}=\right.$ $.0019, p \leq .001]$, A.W.M. $\left[F(5,30)=10.45, M S_{\mathrm{e}}=.0015\right.$, $p \leq .001]$, F.H. $\left[F(5,30)=4.35, M S_{\mathrm{e}}=.0014, p \leq .005\right]$, and A.K. $\left[F(5,30)=14.85, M S_{\mathrm{e}}=.0014, p \leq .001\right]$. These results clearly demonstrate that the matched conditions yielded superior performance across Experiments 1 and 2 .

\section{Discussion}

Experiment 2 was conducted to evaluate whether task demands contributed to the matched advantage observed in Experiment 1. In Experiment 2, we modified the task 

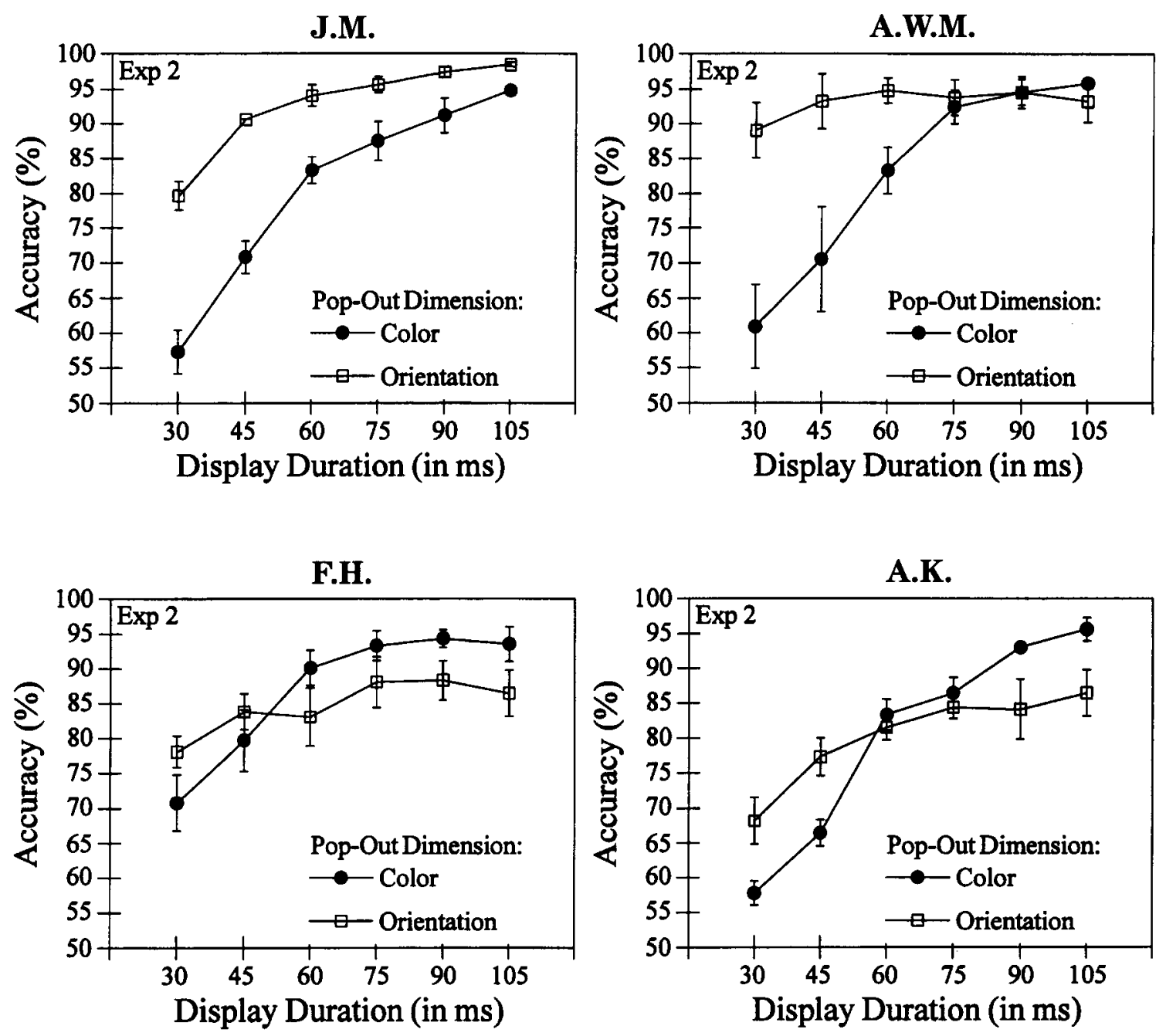

Figure 6. Orientation discrimination accuracy from Experiment 2 for each of the 4 observers as a function of display duration and pop-out dimension.

structure-mixing pop-outs and absolute identification-while maintaining the trial-level relationships among distractors and targets. An object-based account predicted that these changes in the task structure would eliminate the matched advantage. Feature-based models of visual attention, on the other hand, predicted that the matched advantage would remain because the relations among the distractor and target values at the trial level were unchanged from Experiment 1. A matched advantage was indeed observed in Experiment 2, consistent with feature-based accounts of attention, and counter to the predictions implied by an object-based view. ${ }^{1}$

The only statistical difference observed between the results of Experiments 1 and 2 was in the absolute level of performance for 2 of the observers. Observer A.W.M.'s level of performance was lower for Experiment 2 than for Experiment 1, whereas Observer J.M. showed the opposite pattern. These differences in accuracy were probably due to practice effects, because Observer A.W.M. per- formed Experiment 2 first, whereas Observer J.M. performed Experiment 1 first.

Apart from the absolute differences in performance level, the qualitative pattern of results remained relatively constant. In particular, for each observer, the pattern of data in Experiment 2 mirrored that of Experiment 1 . Most important, the interaction between pop-out dimension and discrimination dimension was conspicuous in each experiment. The results are perhaps surprising considering the differences between the two tasks: In Experiment 1, observers knew on a given trial the dimension creating the pop-out, and so observers were free to devote all of their efforts to making discriminations on that dimension; in Experiment 2, there was uncertainty on each trial as to how the target would be defined, and observers were required each time to make discriminations on both dimensions. The replicability of the results across experiments with different task demands lends . strong support to the feature-based explanation, with its 


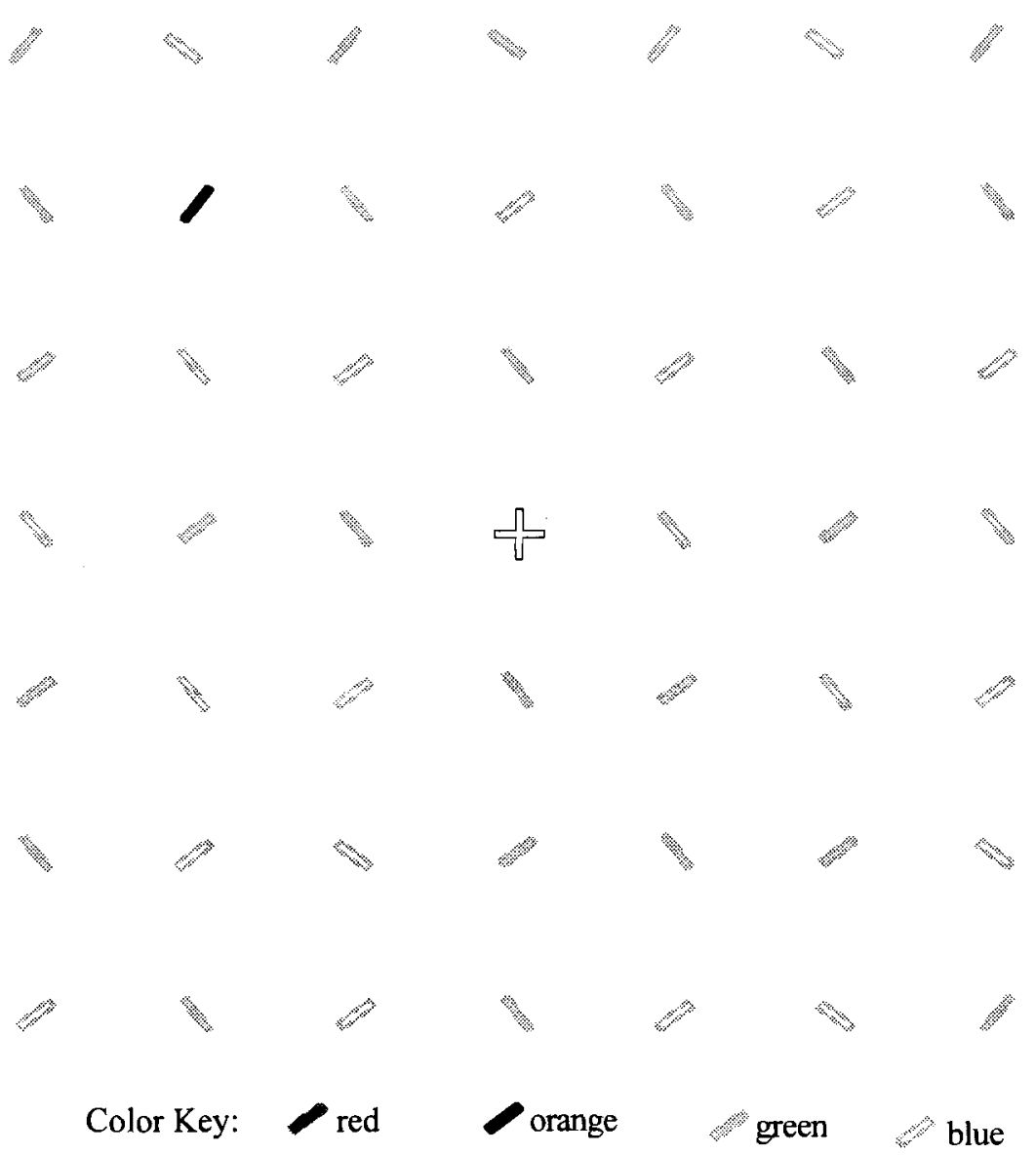

Figure 7. An example of a checker color pop-out stimulus display from Experiment 3.

focus on the stimulus relations within a given display, which was constant across the two experiments. In the final experiment in this study, we explored the impact of these stimulus relations by manipulating the characteristics of the stimulus display.

\section{EXPERIMENT 3}

In Experiments 1 and 2, we extended the standard feature search task: Observers had to locate a target element and make a discrimination on one (Experiment 1 ) or two (Experiment 2) of the target's features. We also manipulated the overlap between the distractor values and the target values, creating situations in which the distractor values were drawn from the target set (mismatched conditions) or differed substantially from the potential target values (matched conditions). Both experiments uncovered a matched advantage (i.e., a performance advantage for matched conditions over mismatched conditions). The results support feature-based models, which state that because the distractor values in the mismatched conditions were drawn from the target set, they were more difficult to exclude from the discrimination process. In the matched conditions, on the other hand, the distractor val- ues had no overlap with the target values, and even if they were mistakenly sampled by attentional mechanisms, they were relatively easy to exclude from the decision process. This explanation was examined in greater detail in Experiment 3.

If the overlap between the set of distractor values and the set of target values caused the relative decrements in performance observed in the mismatched conditions, then reducing the number of distractors that share values with potential targets should improve discrimination performance. This was the logic adopted in the present experiment. A new type of stimulus display was introduced, called the "checker" display. As in the other displays, a unique value on one of the two dimensions - the pop-out dimension-defined the target. However, in the checker display, half of the distractor values on the non-pop-out dimension overlapped with values from the target set, whereas the other half were outside the target set. The values inside and outside the target set alternated in the form of a checkerboard, hence the name of the display (Figure 7).

For a given discrimination dimension, the checker display represents a compromise between the matched conditions, in which there was $0 \%$ value overlap between tar- 


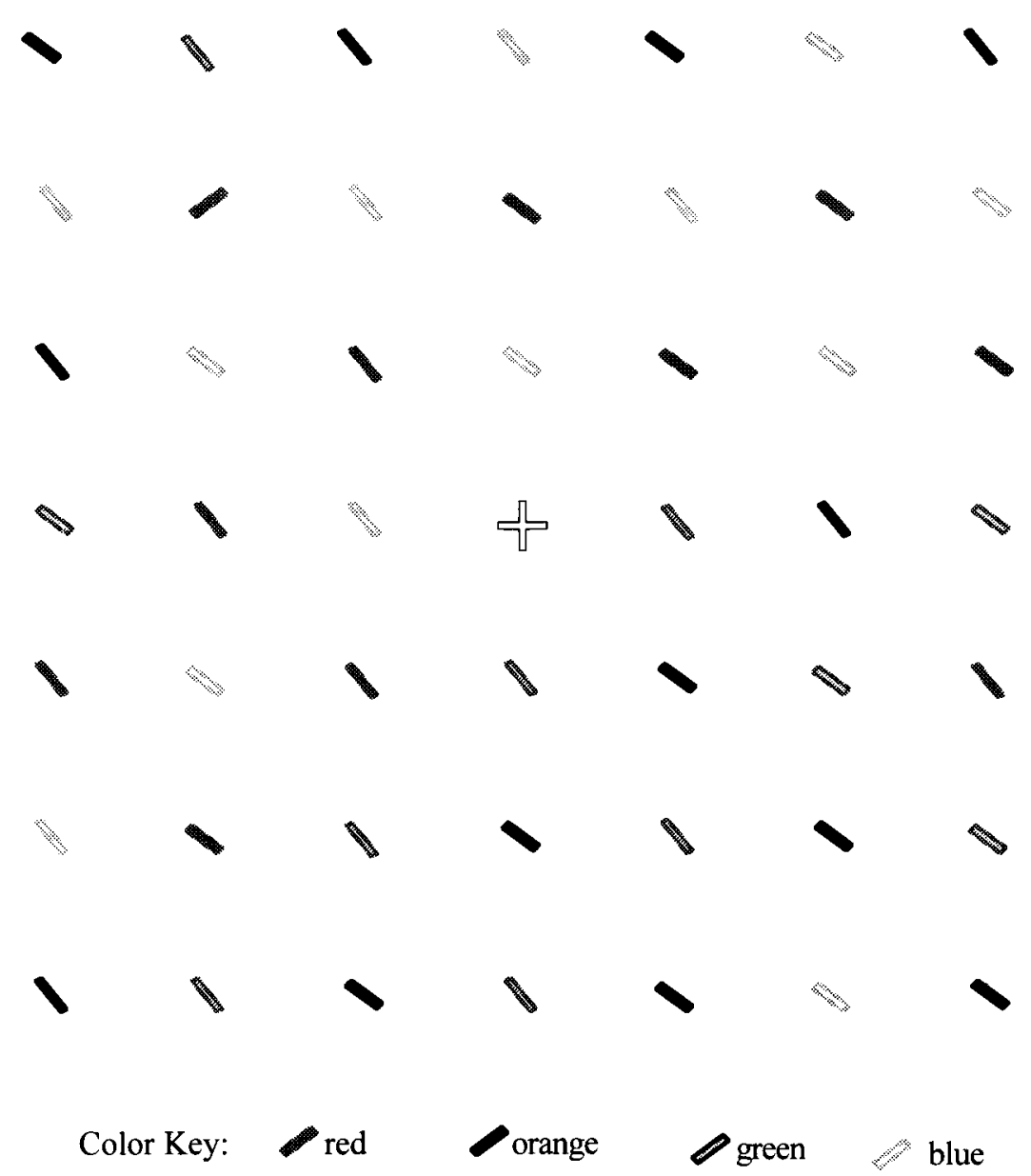

Figure 8. An example of a checker orientation pop-out stimulus display from Experiment 3.

gets and distractors, and the mismatched conditions, in which there was $100 \%$ value overlap between targets and distractors. More specifically, the checker display represents $50 \%$ value overlap between targets and distractors, because only half of the distractors in the checker displays were drawn from the target set. If task interference is a function of the ease with which distractors are excluded from the decision process, then we would expect discrimination accuracy in the $50 \%$ value overlap conditions, in which targets and distractors are moderately confusable, to lie between accuracy in the $0 \%$ value overlap conditions (i.e., matched) and in the $100 \%$ value overlap conditions (i.e., mismatched).

\section{Method}

\section{Observers, Apparatus, and Stimulus Values}

Three of the 4 observers from the previous experiments participated (J.M., A.W.M., and A.K.). The apparatus was identical to those used in the previous three experiments. Trial initiation, presentation, responding, and poststimulus masking occurred as in Experiment 2, which also used the absolute identification task. The two target values for color and orientation from the previous ex- periments were used, as were the two different distractor values for each dimension.

\section{Procedure}

The procedure used and the conditions tested were identical to those of Experiment 2, with the following exceptions: A third type of stimulus display was added (described below), the number of display durations examined was reduced to four $(30,45,60$, and $75 \mathrm{msec}$ ), and the number of trials was increased to four blocks of 320 trials. Display duration was blocked, occurring in a random order for each session. Display types were mixed randomly within each block of trials.

\section{Conditions and Display Types}

As in Experiment 2, the target stimuli were created by combining the two target values for color with the two target values for orientation, yielding four possible targets: orange $/ 38^{\circ}$, orange $/ 52^{\circ}$, $\mathrm{red} / 38^{\circ}$, and red $/ 52^{\circ}$. Responses were obtained as in Experiment 2 . As in the previous experiments, the target item was designated by a unique color or unique orientation value. However, in this experiment, the distractor values on the non-pop-out dimension could assume two different patterns, resulting in the four different display types described below.

Regular color pop-out. The targets were color pop-outs, meaning that one of the four targets was embedded in an array of dis- 
J.M.

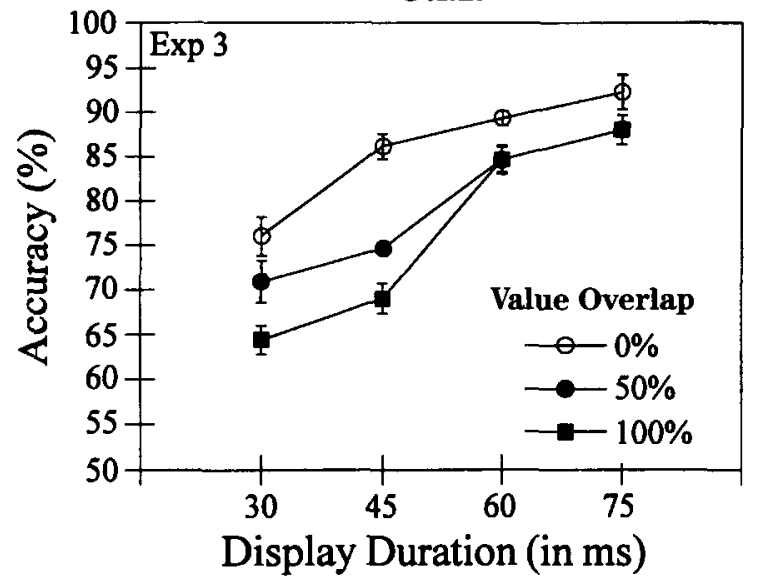

A.W.M.

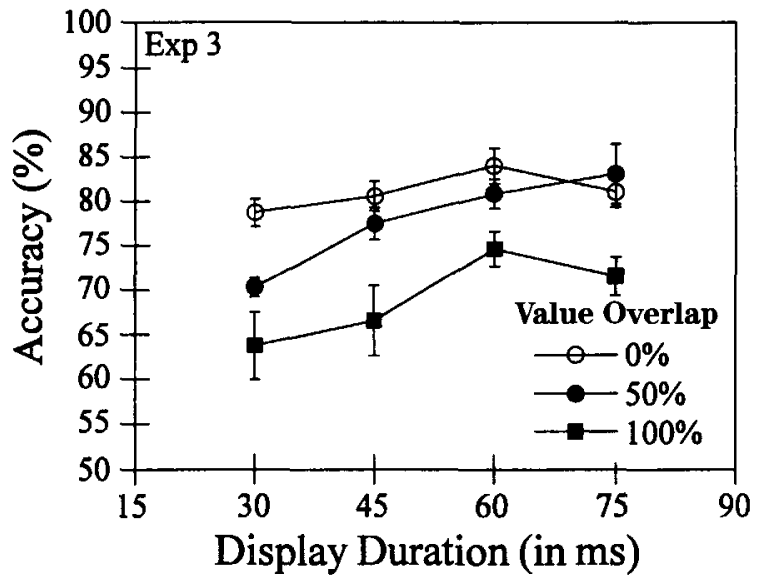

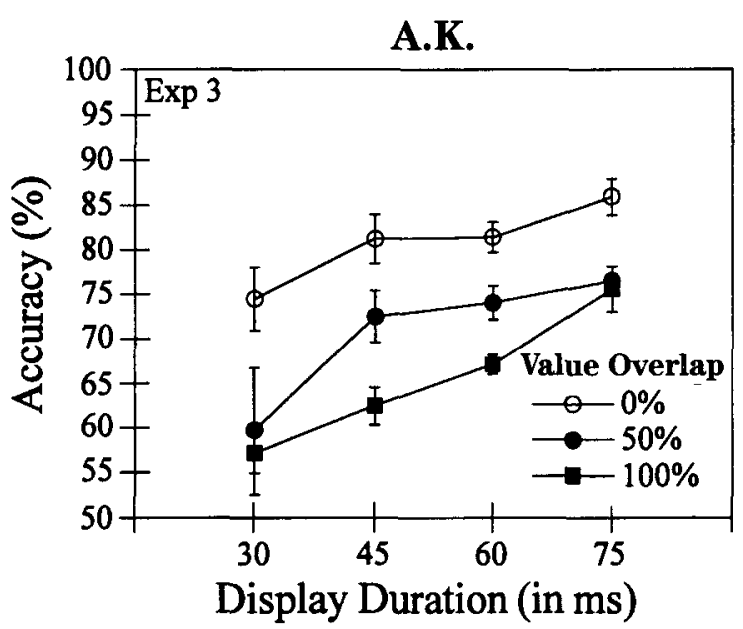

Figure 9. Color discrimination accuracy from Experiment 3 for each of the 3 observers as a function of display duration and value overlap. Open circles represent the $0 \%$ value overlap (color pop-out) displays, solid circles represent the $50 \%$ value overlap (checker orientation pop-out) displays, and solid squares represent the $100 \%$ value overlap (regular orientation popout) displays. Error bars depict $\pm 1 S E$.

tractors, half of which were blue, the other half green. The orientation values assumed by the distractors were either $38^{\circ}$ or $52^{\circ}$. This type of display is identical to the color pop-out displays used in the previous experiments (Figure 1).

Checker color pop-out. Again, targets were color pop-outsone of the four target stimuli was embedded in an array of distractors, half of which were blue, the other half green. However, the orientation of the distractors assumed one of four values: $38^{\circ}, 52^{\circ}$, $308^{\circ}$, or $322^{\circ}$. These orientations were arranged so that every other display element was oriented to the left of vertical, while the remaining elements were oriented to the right of vertical, mimicking a checkerboard arrangement. This type of display is illustrated in Figure 7. Note that, as in the regular color pop-out displays, an observer cannot determine the target on the basis of its orientation.

Regular orientation pop-out. The targets were orientation popouts, meaning that one of the four target stimuli was embedded in an array of distractors, half of which were $308^{\circ}$, the other half $322^{\circ}$. The color values assumed by the distractors were either red or orange. This type of display is identical to the orientation pop-out displays used in the previous experiments (Figure 2).
Checker orientation pop-out. This type of display is analogous to that used in the checker color pop-out trials: Targets were orientation pop-outs, with one of the four target stimuli appearing among distractors with an equal distribution of $308^{\circ}$ and $322^{\circ}$ orientations. Distractors assumed one of four colors: red, orange, blue, or green, with red/orange values alternating with blue/green values. An example of this type of display is illustrated in Figure 8. Again, the location of the target was discernible only via orientation information; the location of the target was ambiguous on the basis of color.

\section{Results}

For each type of discrimination (color or orientation), the data were partitioned according to the proportion of distractor values drawn from the target set: $0 \%, 50 \%$, or $100 \%$. When the pop-out and discrimination dimensions matched, both the regular displays and the checker displays involved a $0 \%$ value overlap between the distractor and target values (matched conditions). When the pop-out 
J.M.

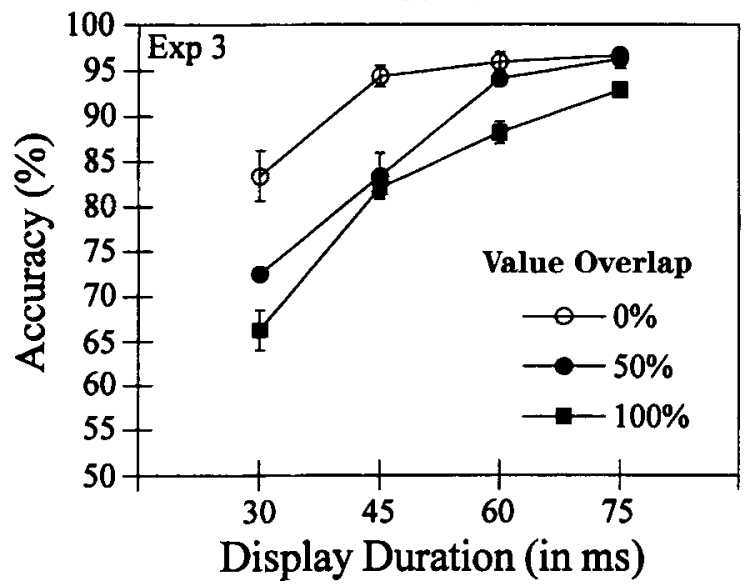

A.W.M.

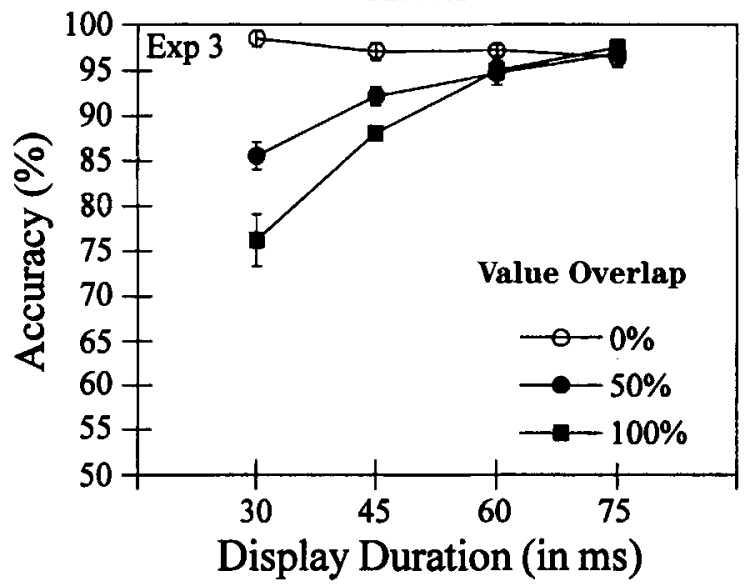

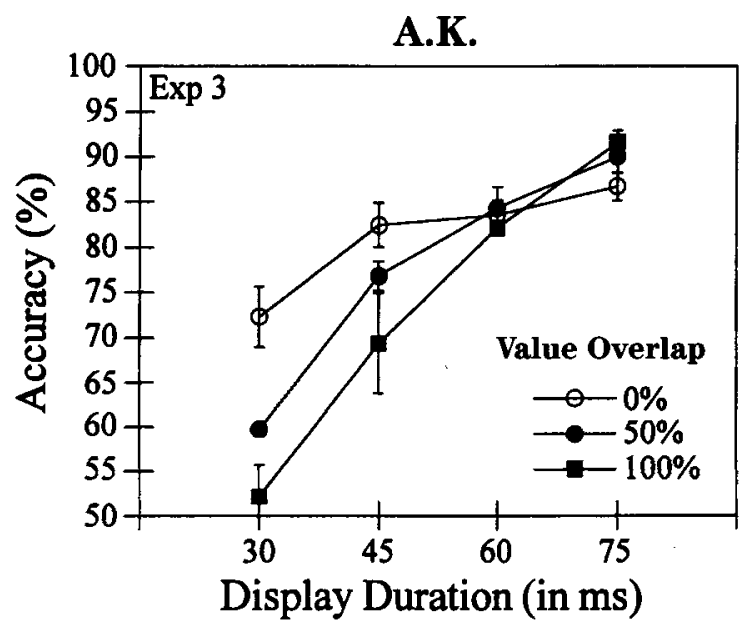

Figure 10. Orientation discrimination accuracy from Experiment 3 for each of the 3 observers as a function of display duration and value overlap. Open circles represent the $0 \%$ value overlap (orientation pop-out) displays, solid circles represent the $50 \%$ value overlap (checker color pop-out) displays, and solid squares represent the $100 \%$ value overlap (regular color pop-out) displays. Error bars depict $\pm 1 S E$.

dimension and discrimination dimensions mismatched, the checker displays involved a $50 \%$ value overlap between distractor and target values, whereas the regular displays involved a $100 \%$ value overlap between distractor and target values (mismatched conditions). The three panels of Figure 9 show the accuracy of color discriminations for each of the 3 observers as a function of value overlap $(0 \%$, $50 \%$, and $100 \%$ ). The three panels of Figure 10 contain analogous information for orientation discriminations.

The data (i.e., proportion correct) were analyzed using a randomized block design with repeated measures, treating observer (three levels) as a random factor and discrimination dimension (two levels), value overlap (three levels), and display duration (four levels) as fixed factors, with session providing the error term for each cell. Main effects of observer $\left[F(2,9)=96.94, M S_{\mathrm{e}}=.0028, p \leq\right.$ $.001]$, value overlap $\left[F(2,4)=117.17, M S_{\mathrm{e}}=.0022, p<\right.$
$.001]$, and display duration $\left[F(3,6)=18.73, M S_{\mathrm{e}}=.0214\right.$, $p<.005]$ proved reliable. Also reliable were the two-way interactions between observer and discrimination dimension $\left[F(2,9)=120.31, M S_{\mathrm{e}}=.0008, p<.001\right]$, observer and display duration $\left[F(6,27)=7.14, M S_{\mathrm{e}}=.0030, p<\right.$ $.001]$, value overlap and display duration $[F(6,12)=13.37$, $\left.M S_{\mathrm{e}}=.0019, p<.001\right]$, and the three-way interactions involving observer, discrimination dimension, and value overlap $\left[F(4,18)=4.92, M S_{\mathrm{e}}=.0013, p<.01\right]$; observer, discrimination dimension, and display duration $[F(6,27)=$ $\left.3.68, M S_{\mathrm{e}}=.0017, p<.01\right]$; and discrimination dimension, value overlap, and display duration $[F(6,12)=3.74$, $\left.M S_{\mathrm{e}}=.0016, p<.05\right]$.

Examination of Figures 9 and 10 reveals that, for all 3 observers, performance in the $50 \%$ value overlap conditions was intermediate to that of the $0 \%$ value overlap conditions and the $100 \%$ value overlap conditions for both 
color and orientation discriminations. To corroborate this observation, planned comparisons among these conditions were conducted separately for color and orientation discrimination accuracies (collapsed across observer and display duration). The analyses of color discriminations showed that performance for the $50 \%$ value overlap condition (i.e., checker orientation pop-out) lagged behind performance for the $0 \%$ value overlap conditions (i.e., regular and checker color pop-out) $[F(1,9)=90.92$, $\left.M S_{\mathrm{e}}=.0011, p<.001\right]$, yet was superior to $100 \%$ value overlap conditions (i.e., regular orientation pop-out) $\left[F(1,9)=21.85, M S_{\mathrm{e}}=.0035, p<.005\right]$. Analyses of orientation discriminations revealed a similar pattern: Performance on the $0 \%$ value overlap conditions (i.e., regular and checker orientation pop-out) outpaced performance on the $50 \%$ value overlap condition (i.e., checker color pop-out) $\left[F(1,9)=66.75, M S_{\mathrm{e}}=.0008, p<.001\right]$, which, in turn, differed reliably from the $100 \%$ value overlap conditions (i.e., regular color pop-out) $[F(1,9)=43.53$, $\left.M S_{\mathrm{e}}=.0008, p<.001\right]$. Planned comparisons on individual observers' data revealed similar results.

\section{Discussion}

The results from Experiment 3 again reveal a matched advantage, as was found in the first two experiments. This experiment explored the nature of this advantage by examining the role of target-distractor overlap. Specifically, we manipulated the proportion of distractors possessing potential target values. If performance in the mismatched tasks in the previous experiments suffered because the distractors were confusable with target values, then decreasing the overlap between distractors and targets should produce an increase in performance. Results are in clear agreement with this prediction. The $0 \%$ value overlap conditions (analogous to the matched conditions of Experiments 1 and 2) yielded better performance than did the $100 \%$ value overlap conditions (analogous to the mismatched conditions of Experiments 1 and 2), with performance in the $50 \%$ value overlap falling between that of these two conditions. These results support our explanation that the matched advantage is rooted in confusion produced by distractors in the mismatched conditions, as implied by feature-based models of attentional selection.

\section{GENERAL DISCUSSION}

\section{Summary}

Three experiments explored the nature of visual selective attention. Specifically, we asked whether attentional selection is best characterized as operating in the feature or object domain. A comparison between featurebased and object-based models was made by (1) extending the requirements of the typical search task to include the discrimination of target values and (2) manipulating the similarity of distractors to targets and examining its influence on target discriminations. In our matched conditions, the target's defining feature (i.e., the feature that led to pop-out) was also the feature to be discriminated, thereby keeping the distractor values distinct from potential target values. In our mismatched conditions, the target-defining and discrimination dimensions differed; here distractor values were drawn from the set of possible target values, making it impossible to differentiate targets from distractors on the basis of the discrimination dimension alone.

We conceptualized feature-based attentional selection as sampling information from a given spatial region for further processing and decision making (see, e.g., Logan, 1996). On this view, it was predicted that limiting the processing time would hinder discrimination performance. Specifically, we assumed that the sampling of information from distractor locations would hamper the identification process. The extent to which this added noise disrupted the discrimination of the target values was thought to depend upon the confusability of targets and distractors. In the matched conditions, confusability was relatively low, so distractor values could presumably be filtered or excluded from decision processes. By contrast, in the mismatched conditions, confusability was relatively high; indeed, filtering based on feature values was impossible in the mismatched conditions. Feature-based models thus predicted poorer performance in the mismatched conditions relative to the matched conditions.

From the object-based perspective, we assumed that attention selected fully integrated objects (i.e., all features associated with an object) into a short-term memory system. Once selected, the objects could inform response processes. Consistent with Duncan and Humphreys's (1989) AET, objects were assumed to be perceptual units with a strong grouping of all constituent featuressuccessful selection of one feature of an object thus implied the successful selection of all features of that object. Similarly, if attention failed to select the target object, none of the target features would be available to inform the response. According to this view, as long as target selection occurs successfully and efficiently, it is irrelevant which feature drives the selection process. Thus, according to object-based models, no differences were predicted between the matched and mismatched conditions. ${ }^{2}$

Consistent with the predictions of feature-based models, Experiment 1 revealed a performance advantage for the matched conditions. Experiment 2 replicated these results in a modified experimental task, which ruled out object-based accounts linked to task demands. Finally, Experiment 3 demonstrated that it was the degree of overlap between distractor and target values that modulated performance in this task.

\section{Models of Attentional Selection}

\section{Feature Models}

As described throughout the paper, the results from these three experiments are consistent with attentional selection in the feature domain. In the space-based models of feature selection, attention selects a region of space 
and consequently all features occupying the selected region. We used Logan's (1996) CTVA as a model representative of this view.

Logan's (1996) CODE-TVA couples Bundesen's (1990) TVA with van Oeffelen and Vos's $(1982,1983)$ contour detector theory (CODE). Logan engaged Bundesen's TVA in filtering and categorizing information sampled by a spatial selection mechanism that is driven by a CODE description of the visual scene. CODE-TVA provides a coherent account of results from the present study because, under certain conditions, the system (1) may sample information from irrelevant locations, which may explain the relatively poor accuracy observed in the mismatched conditions; and (2) can filter out irrelevant information on the basis of selected feature values, which may explain the relatively good performance observed in the matched conditions.

Let us first consider sampling information from irrelevant objects. Logan (1996) proposed that the selection mechanism samples information from a spatially welldefined region, defined by the CODE module. Although the sampling mechanism is spatially precise, the feature representations of objects are distributed in space. As a result, featural representations of neighboring objects may overlap. When attention is directed to a specific location in space, the featural information sampled will predominantly belong to the object at that location, but may also include featural information from neighboring objects.

The sampled information feeds into the TVA module, where a competitive process yields a categorization response. As described in Bundesen's (1990) original theory, the categorization process is controlled by featurespecific attentional weights determined by the task demands. Thus, feature values that are task irrelevant, such as distractor values in the matched conditions, may be filtered early from the competition because their associated attentional weight is small. By contrast, distractor feature values in our mismatched conditions were also potential target values; hence, presumably their associated attentional weights were comparatively large. Increased competition can slow categorization of target values, resulting in decreased accuracy for brief exposures, as was found in the present study. Further, by reducing the number of task-relevant distractor values, as we did in Experiment 3 , we effectively reduced response competition, yielding a predicted (and observed) increase in accuracy. Thus, Logan's (1996) CTVA is consistent with the present set of data because it combines spatial imprecision in selecting features with postselection filtering. However, as we will see, it is also possible to handle our data with a class of model based in a very different architecture.

\section{Hybrid Feature Selection}

In our realizations of the feature-based and objectbased accounts, we assumed a constant rate of selection on both feature dimensions. For example, in the featurebased models, orientation information was sampled at the same rate as color information, regardless of the targetdefining feature. Likewise, in the object-based accounts, we assumed that in the selection of an object, its color and orientation values became available simultaneously. Indeed, the failure of object-based models shown in the present study can be traced directly to this assumption, because our results show that levels of performance differed across the different features of an object. Relaxing the assumption of a constant selection rate yields a class of models consistent with our results. Specifically, these alternative models allow for attentional selection to occur at different rates on each dimension, depending upon the dimension defining the target.

In describing AET, Duncan and Humphreys (1989) proposed that homogeneous distractors that are distinct from the target are inhibited en masse, resulting in the efficient selection of the target object. Such inhibition was thought to operate at the level of fully integrated objects. Let us assume instead that such a mechanism operates at the level of individual feature maps. Then, within a specific feature map, distractor representations could be inhibited when a target feature is unique (e.g., in the matched conditions), but not when the target value is similar to its distractors (mismatched conditions). Selection of target features in the mismatched conditions would need to wait for input from the feature map containing the target-defining feature. Hence, selection of the target representation from the response-relevant feature map would occur more slowly in mismatched conditions than in matched conditions. Several models with an architecture of this type have been introduced recently (e.g., Duncan, 1996; Houghton \& Tipper, 1994; Schneider, 1995).

One example is Duncan's (1996) integrated competition hypothesis, mentioned briefly in the present introduction. In this model, distinct processing centers, corresponding to the different aspects of an object, select features through a competitive process. Integrated links among the various features of each object ensure that, ultimately, the features that win the competition within each center correspond to a single object. Duncan's (1996) approach can accommodate the results of the present study if we assume separate processing centers for color and orientation (or form) with weighting within each center being a function of task relevance (e.g., name color vs. name orientation). In our matched conditions, the target feature was the only task-relevant element in the display, so it would readily win the competition with the other elements within that center. By contrast, in the mismatched conditions, all objects within the processing center coding the decision feature were task relevant. Thus, both target and distractor features would be accorded hefty attentional weights, thereby conferring no selection advantage to the targets relative to the distractors within this system. Correct selection of the target object in the mismatched conditions would thus depend upon input from another center. Therefore, even though the target feature representation would eventually win the competition within its center, because target selection in the mismatched conditions must await input from a separate center, performance in these conditions would suffer relative to performance in the matched conditions. 
The integrated competition hypothesis is also consistent with the results from our Experiment 3, in which we varied the proportion of distractors that could compete with target selection in the mismatched conditions. Presumably, by reducing the number of task-relevant features, we reduced the competition for selection, enabling target selection within the target's processing center to occur relatively more efficiently.

As the foregoing discussion makes clear, then, both Logan's (1996) and Duncan's (1996) models of visual selective attention are consistent with the results of the present study. Although they are functionally different, these models are similar in that they allow for separate processing of the features of an object. At later stages in perceptual processing, these models allow the features of an object to be strongly grouped and operated upon as a single entity. However, at least initially in processing, features appear to be processed in an independent fashion in which the processing benefits that accrue to one aspect of an object do not immediately benefit all aspects of that object. In other words, the perceptual context created with each dimension can have a powerful effect on attentional selection, and our data suggest that these contextual effects operate initially at the feature level rather than at the object level.

\section{Relation to Flanker Studies}

In the present study, we borrowed the logic adopted in the flanker compatibility studies popularized by Eriksen and colleagues (e.g., B.A. Eriksen \& C.W. Eriksen, 1974). Here a target stimulus is flanked by distractors whose identity is either compatible or incompatible with the response to the target. The standard finding in this literature is that incompatible distractors slow decisions to the target, whereas compatible distractors speed decisions, relative to response-neutral flanking letters. In flanker experiments, the target's defining feature is typically its location (i.e., the target is the center element in a string of three or five elements), while the to-be-reported feature is shape (e.g., letter identity). According to the present analysis, there is a mismatch between the target's defining feature (location) and the feature to be discriminated (e.g., letter identity). As a result, selecting the taskrelevant target features (e.g., the target's shape), while excluding irrelevant flanker features (e.g., the flankers' shapes), should be difficult in this situation. ${ }^{3}$

One important difference should be noted between the typical flanker study and our study. In the standard flanker task, responses are speeded, and reaction time is the primary measure. Most explanations of the flanker compatibility effect hence attribute the interference to difficulties in response selection, with responses being slowed due to the activation of competing motor responses (e.g., Coles, Gratton, Bashore, Eriksen, \& Donchin, 1985; C.W. Eriksen \& Schultz, 1979). In the present task, we used brief exposures and looked at unspeeded accuracies. According to Santee and Egeth (1982), accuracy to brief exposures often yields different results from reaction times to response-terminated displays, even though the paradigm may be identical. These authors argued that accuracy is more sensitive to perceptual interference, whereas reaction time is more sensitive to interference in response selection. Our finding of flanker-compatibility-like effects in the present study most probably reflects perceptual decision processes, because our task was data limited and unspeeded. This suggests either that the flanker task taps different processes from those tapped by the present task, or that interference in flanker-style tasks also occurs at a perceptual or decisional level (see, e.g., Botella, 1996).

\section{Conclusion}

Many recent studies have supported the view that visual selective attention is coordinated at the level of objects (e.g., Driver \& Baylis, 1989; Duncan, 1984, 1993; Kramer \& Jacobson, 1991; Kramer \& Watson, 1996; Tipper, Brehaut, \& Driver, 1990; Tipper, Driver, \& Weaver, 1991; Umiltà, Castiello, Fontana, \& Vestri, 1995) rather than at the level of features in visual space (but see C.W. Eriksen \& St. James, 1986; Kim \& Cave, 1995; LaBerge \& Brown, 1989; Posner, Snyder, \& Davidson, 1980). Object-based selection is typically viewed as occurring late in perceptual processing, after the integration of the various features of an object (see, e.g., Duncan \& Humphreys, 1989, 1992). Yet, regardless of how the linkages among features of an object are set up (common location vs. common object token), our results suggest that attentional selection is coordinated at the level of features (see also Lavie, 1997). Depending on the context, accurate information may become available about certain features before others. In the present study, the distinctiveness of an object's features within the visual array was the basis of these differences in featural processing. Our results are consistent with recent models of attentional selection that allow for the partially independent selection or processing of the features of an object (e.g., Duncan, 1996; Logan, 1996).

\section{REFERENCES}

Botella, J. (1996). Decision competition and response competition: Two main factors in the flanker compatibility effect. In A. F. Kramer, M. G. H. Coles, \& G. D. Logan (Eds.), Converging operations in the study of visual selective attention (pp. 503-518). Washington, DC: American Psychological Association.

BUNDESEN, C. (1990). A theory of visual attention. Psychological Review, 97, 523-547.

CAVE, K. R., \& WolfE, J. M. (1990). Modeling the role of parallel processing in visual search. Cognitive Psychology, 22, 225-271.

Coles, M. G. H., Gratton, G., Bashore, T. R., Eriksen, C. W., \& DonCHIN, E. (1985). A psychophysical investigation of the continuous flow model of human information processing. Journal of Experimental Psychology: Human Perception \& Performance, 11, 529-553.

Compton, B. J., \& LoGAN, G. D. (1993). Evaluating a computational model of perceptual grouping by proximity. Perception \& Psychophysics, 53, 403-421.

Driver, J., \& BAYLIS, G. C. (1989). Movement and visual attention: The spotlight metaphor breaks down. Journal of Experimental Psychology: Human Perception \& Performance, 15, 448-456

DUNCAN, J. (1984). Selective attention and the organization of visual information. Journal of Experimental Psychology: General, 113, 501517. 
DUNCAN, J. (1989). Boundary conditions on parallel processing in human vision. Perception, 18, 457-469.

DUNCAN, J. (1993). Similarity between concurrent visual discriminations: Dimensions and objects. Perception \& Psychophysics, 54, 425430.

DUNCAN, J. (1996). Cooperating brain systems in selective perception and action. In T. Inui and J. L. McClelland (Eds.), Attention and performance XVI: Information integration in perception and communication (pp. 549-578). Cambridge, MA: MIT Press.

DunCAN, J., \& Humphreys, G. W. (1989). Visual search and stimulus similarity. Psychological Review, 96, 433-458.

DUNCAN, J., \& HumpHrEYs, G. W. (1992). Beyond the search surface: Visual search and attentional engagement. Journal of Experimental Psychology: Human Perception \& Performance, 18, 578-588.

ERIKSEN, B. A., \& ERIKSEN, C. W. (1974). Effects of noise letters upon the identification of a target letter in a nonsearch task. Perception $\&$ Psychophysics, 16, 143-149.

ERIKSEN, C. W., \& SChUlTZ, D. W. (1979). Information processing in visual search: A continuous flow conception and experimental results. Perception \& Psychophysics, 25, 249-263.

ERIKSEN, C. W., \& ST. JAMES, J. D. (1986). Visual attention within and around the field of focal attention: A zoom lens model. Perception \& Psychophysics, 40, 225-240.

GolDSMITH, M. (1998). What's in a location? Comparing object-based and space-based models of feature integration in visual search. Journal of Experimental Psychology: General, 127, 189-219.

Houghton, G., \& TIPPER, S. P. (1994). A model of inhibitory mechanisms in selective attention. In D. Dagenbach \& T. H. Carr (Eds.), Inhibitory processes in attention, memory, and language (pp. 53-112). New York: Academic Press.

Kamneman, D., Treisman, A., \& GibBs, B. (1992). The reviewing of object files: Object-specific integration of information. Cognitive Psychology, 24, 175-219.

KANWISHER, N., \& DrIVER, J. (1992). Objects, attributes, and visual attention: Which, what, and where. Psychological Science, 1, 26-31.

KIM, M.-S., \& CAVE, K. R. (1995). Spatial attention in visual search for features and feature conjunctions. Psychological Science, 6, 376-380.

Koch, C. \& Ullman, S. (1985). Shifts in selective visual attention: Towards the underlying neural circuitry. Human Neurobiology, 4, 219 227.

Kramer, A. F., \& JACOBSON, A. (1991). Perceptual organization and focused attention: The role of objects and proximity in visual processing. Perception \& Psychophysics, 50, 267-284

Kramer, A. F., \& Watson, S. E. (1996). Object-based visual selection and the principle of uniform connectedness. In A. F. Kramer, M. G. H. Coles, \& G. D. Logan (Eds.), Converging operations in the study of visual selective attention (pp. 395-414). Washington: American Psychological Association.

LABERGE, D., \& Brown, V. (1989). Theory of attentional operations in shape identification. Psychological Review, 96, 101-124.

LAVIE, N. (1997). Visual feature integration and focused attention: $\operatorname{Re}-$ sponse competition from multiple distractor features. Perception \& Psychophysics, 59, 543-556.

LoGAN, G. D. (1996). The CODE theory of visual attention: An integration of space-based and object-based attention. Psychological Review, 103, 603-649.

NOTHDURFT, H.-C. (1992). Feature analysis and the role of similarity in preattentive vision. Perception \& Psychophysics, 52, 355-375.

NothdurfT, H.-C. (1993). The role of features in preattentive vision: Comparison of orientation, motion, and color cues. Vision Research, 33, 1937-1958.

Posner, M. I., SNyder, C. R. R., \& DavidSON, B. J. (1980). Attention and the detection of signals. Journal of Experimental Psychology: General. 109, 160-174

SANTEE, J. L., \& Egeth, H. E. (1982). Do reaction time and accuracy measure the same aspects of letter recognition? Journal of Experimental Psychology: Human Perception \& Performance, 8, 489-501.
SCHNEIDER, W. X. (1995). VAM: A neuro-cognitive model for visual attention control of segmentation, object recognition, and space-based motor action. Visual Cognition, 2, 331-375.

Tipper, S. P., Brehaut, J. C., \& Driver, J. S. (1990). Selection of moving and static objects for the control of spatially-directed action. Journal of Experimental Psychology: Human Perception \& Performance, 16, 492-504.

Tipper, S. P., Driver, J. S., \& Weaver, B. (1991). Object-centered inhibition of return of visual attention. Quarterly Journal of Experimental Psychology, 43A, 289-298.

Treisman, A. M. (1988). Features and objects: The fourteenth Bartlett memorial lecture. Quarterly Journal of Experimental Psychology, 40, 201-237.

Treisman, A. M. (1993). The perception of features and objects. In A. D. Baddeley \& L. Weiskrantz (Eds.), Attention: Selection, awareness, and control (pp. 5-35). Oxford: Clarendon.

Umiltà, C., Castiello, U., Fontana, M., \& Vestri, A. (1995). Objectcentred orienting of attention. Visual Cognition, 2, 165-181.

van Oeffelen, M. P., \& Vos, P. G. (1982). Configurational effects on the enumeration of dots: Counting by groups. Memory \& Cognition, 10, 396-404

van Oeffelen, M. P., \& Vos, P. G. (1983). An algorithm for pattern description on the level of relative proximity. Pattern Recognition, 16, 341-348.

WINER, B. J. (1971). Statistical principles in experimental design (2nd ed.). New York: McGraw-Hill.

\section{NOTES}

1. An alternative explanation that was also ruled out by Experiment 2 is that observers' preparations to make discriminations affected the selection rate. In Experiment 1, preparing to make a color discrimination might have sped the selection of targets defined by color, but not orientation. This also would have produced the matched advantage observed in Experiment 1. However, this explanation would predict the absence of the matched advantage in Experiment 2, since observers prepared to make both color and orientation discriminations on every trial.

2. One reviewer suggested that the present pattern of results can be explained in terms of AET if we assume that with short exposure durations, distractor items enter into VSTM along with the target. This assumption is permissible because, according to Duncan and Humphreys (1989), the capacity of VSTM is not restricted to a single item. However, to account for the matched advantage, one must further assume that features from the distractors that enter VSTM (especially on the mismatched dimension) can interfere with target discriminations. Unfortunately, this further assumption implies differential processing of features within VSTM, an idea that runs counter to a basic tenet of AET - namely, that attention plays no role in perceptual processing: AET states unambiguously that features are processed to the categorical level prior to entry into VSTM as parts of structural units (see, e.g., Duncan \& Humphreys, 1989 , p. 445)

If we reject this tenet and suppose instead that features undergo differential processing within VSTM, in essence we reconceptualize selection as feature based because the different features of an object are being represented and processed separately from one another. The only difference, then, between this type of model and more typical featurebased models is that here selection is operating in a restricted set of items that have gained entry into VSTM, rather than in all elements in the visual array. We are uncertain about what the added assumption of entry into VSTM offers feature-based models.

3. However, adding a second selection cue such as color (Kramer \& Jacobson, 1991) or motion (Driver \& Baylis, 1989) reduces interference from flankers not possessing this additional target-defining feature.

(Manuscript received August 15, 1997; revision accepted for publication January 29, 1998.) 